
\title{
25 Research Soure \\ Exploring Differential Exon Usage via Short- and Long-read RNA Sequencing Strategies
}

Dena Leshkowitz ( $\nabla$ dena.leshkowitz@weizmann.ac.il)

Weizmann Institute of Science

Merav Kedmi

Weizmann Institute of Science

Yael Fried

Weizmann Institute of Science

David Pilzer

Weizmann Institute of Science

Hadas Keren-Shaul

Weizmann Institute of Science

Elena Ainbinder

Weizmann Institute of Science

Bareket Dassa

Weizmann Institute of Science

\section{Research Article}

Keywords: Alternative splicing, Differential splicing, Differential exon usage, transcriptome, long-reads, short-reads, sequencing, embryonic stem cell, isoform, RNA-Seq

Posted Date: March 7th, 2022

DOI: https://doi.org/10.21203/rs.3.rs-1368550/v1

License: (c) (i) This work is licensed under a Creative Commons Attribution 4.0 International License.

Read Full License

Version of Record: A version of this preprint was published at Open Biology on September 28th, 2022. See the published version at https://doi.org/10.1098/rsob.220206. 


\section{Abstract}

\section{Background}

Alternative splicing is a post-transcriptional biological process that produces various messenger RNAs, and thereby various protein products, from one gene, impacting a wide range of cellular activities and disease processes. However, the accurate reconstruction and quantification of full-length transcripts using short-reads is limited, due to their length. Recent long-reads sequencing technologies may provide a solution by sequencing full-length transcripts, yet to address their higher error rates and lower yields, dedicated analysis tools are being developed and assessed. We wanted to explore the use of both short reads and long Oxford Nanopore Technology (ONT) RNA-Seq reads, with different strategies and tools, for detecting global alternative splicing changes, namely differential splicing (DS), during embryonic stem cell differentiation. Specifically, we used two major strategies for DS analysis: isoform-based and countbased. Isoform based requires a complete and accurate isoform construction and quantification of fulllength transcripts as the basis for a confident DS analysis. Alternatively, count-based strategy uses count units, such as exonic regions or junction regions to detect differential exon usage. Exon-based methods, when utilized with short reads, perform better than the isoform-based methods, however, the benefits of applying this method on long reads are yet to be evaluated.

\section{Results}

Bioinformatics analysis of three platforms, Illumina short reads and two long ONT sequencing technologies, namely cDNA and Direct RNA, revealed the strongest gene-level similarity compared to exon-based and isoform-based. Furthermore, the exon-based strategy was advantageous for long reads in detecting differential exon usage (DEU) at the $3^{\prime}$ UTRs, whereas short reads were advantageous in overall detecting more DEUs. Specifically, many genes with DEUs were discovered, mostly in a platform depended manner. Of them, exons within 20 genes, detected in one or more platforms, were validated by PCR, including key differentiation genes, such as Mdb3 and Aplp1.

\section{Conclusions}

We demonstrate that the exon count-based strategy is also applicable and reliable for long reads. Furthermore, we demonstrate that the tested sequencing platforms complement one another in the detection of DEUs, thus enabling novel biological insights. Specifically, we provide an important sequence and analysis resource for discovering transcriptome changes occurring during stem cell development.

\section{Background}

High-throughput short-read sequencing transcriptional profiling ("RNA-Seq") was pioneered in 2008, enabling quantitative transcriptome-wide surveys of gene expression and alternative splicing [1-4]. RNASeq has greatly expanded our knowledge of the transcriptome, providing reliable quantification and detection of differential expression at the gene level $[5,6]$. However, transcript level or isoform-based 
analysis is error-prone, since short reads cannot unambiguously resolve the connectivity between distant exons, particularly when alternative splicing generates multiple, partially redundant isoforms [6-9]. Isoform-based analysis requires a complete and accurate isoform construction and quantification of fulllength transcripts as the basis for a confident differential splicing (DS) analysis. With the emergence of third-generation sequencing, it is now possible to sequence full-length transcripts "in one go" and directly identifying isoform structures thereby overcoming the challenges posed by computational assembly of short reads [10-13]. Oxford Nanopore Technology (ONT) directly sequences a native single-stranded DNA molecule, by measuring characteristic current changes as the bases are threaded through the nanopore by a molecular motor protein [14]. Using ONT technology, both cDNA and Direct RNA long reads can be sequenced [10-12, 15-19]. In the Direct RNA approach, individual poly-adenylated RNA transcripts are directly sequenced, without recoding and amplification biases inherent in other sequencing methodologies. Yet, the relatively high long reads error rates, of above $10 \%$ for both direct RNA and cDNA sequences, complicate the detection of the transcript's exact exon structure [10,11, 20, 21]. Several computational methods of reference-guided assembly and quantification from long-read sequences have been developed to overcome this challenge [22-24]. Recently, studies have compared the transcriptome landscape between these long- and short-read sequencing technologies [10,11, 13, 15, 16, 25-28]. A study by Mehmood et al. utilizing short reads has shown that exon-based methods generally performed better than the isoform-based methods [9]. However, only a few studies have utilized long reads using the exonbased approach [28-31]. To characterize the strengths and remaining challenges in using long-read approaches, a community effort called the Long-read RNA-Seq Genome Annotation Assessment Project (LRGASP) Consortium has been launched [32].

Differentiation of embryonic stem cells (ESCs) is among the most dynamic processes in biology. Mouse ESCs, derived from the inner cell mass of mouse blastocysts, are pluripotent cells that have the capacity to differentiate into cell types of all three primary germ layers [33]. Regulation of ESC development, pluripotency and reprogramming is mediated by transcription factors [34], and involves transcriptome changes and isoform switching via alternative splicing [35-40]. Recently, long reads were used to study alternative splicing events during early embryogenesis [10, 41].

Here, we characterized the strengths and potential of both short- and long-read sequencing platforms to explore transcriptomic changes during in vitro differentiation of mouse ESCs induced by retinoic acid (RA) $[42,43]$. RNA was collected before and after RA-induced differentiation, and sequenced via Illumina to generate short reads (RNA-Seq). In parallel, long-read sequencing was performed using ONT technology, generating both CDNA and Direct RNA long reads. This is the first bioinformatics study to analyse transcriptome mouse ESCs changes using sequences from three platforms, Illumina short reads and two long ONT sequencing technologies, namely cDNA and Direct RNA, driven to detect changes at the gene, transcript i.e., isoform-based, and exon count-based levels. We observed high correlation between the sequencing technologies at gene expression levels and a lower correlation at the transcript level, in agreement with previous studies [11]. We report significant sequence differences in genomic features between the platforms. In addition, we observed a lower correlation on the exon expression levels than that on the gene level and that most of the differential exon usages (DEUs) were 
detected in a platform dependent fashion. This study demonstrates the importance of using different sequencing technologies, and provides an important resource for interpreting transcriptome changes occurring during ESC development.

\section{Results}

\section{Study design and data processing}

This study focused on detecting transcriptome changes during ESC differentiation. Towards this aim, total mouse RNA was extracted from embryoid body (EB) duplicate samples before (Undiff) and after (Day4) differentiation with RA (Fig. 1A, and Methods). RA plays multiple roles in the nervous system, including induction of neural differentiation, axon outgrowth and neural patterning [42]. The RNA was used for short-read sequencing with the TruSeq library and Illumina platform, and for ONT long-read sequencing technology with both cDNA and Direct RNA kits (named herein as platforms: Illumina TruSeq, ONT cDNA and ONT Direct RNA, respectively). The yield of short reads was around 50M (paired-end fragment sequenced) per sample. The ONT MinION flowcell yield was around 3.5M for CDNA, and around $1 \mathrm{M}$ for the Direct RNA (Table 1). ONT and Illumina dataset sequence processing required different bioinformatics tools, as demonstrated in Fig. 1B and described in the methods section. Analyses starting at the stage of the mapped reads from all three platforms (Illumina TruSeq, ONT cDNA and ONT Direct RNA) were conducted using the same procedures, to compare sequence quality features, and detected expression at the gene, transcript and exon levels, as well as the ability to detect differentially expressed genes (DEGs) and differential exon usages (DEUs).

\section{Inter-platform comparison of aligned read characteristics}

Using the aligned reads, we compared the general sequence quality features between the platforms. The median read length in the ONT Direct RNA platform $(1,615$ bases) was longer than the ONT cDNA $(1,060)$ (Table 1). ONT Direct RNA reads were also found to be longer in the study of Workman et al. [20]. The average ONT error rate for the aligned reads was high, i.e., $11.6 \%$ and $14.3 \%$ in the ONT Direct RNA platform and in the ONT cDNA, respectively, in comparison to that of Illumina (0.3\%), similar to previous reports $[10,11,20,21]$. A difference in the GC content distribution was observed (Fig. 2A and Supplementary Fig. 1). Illumina TruSeq reads exhibited a broader distribution (SD of 8.8) than the ONT reads, and broader than all GENCODE annotated transcripts (SD 6.2-6.4). In addition, all platforms showed a right-shift toward higher GC\% values (48-50\%), compared to that computed for all known transcripts (45\%). To monitor whether ONT captures more novel transcripts, we examined the saturation of known and novel junctions (Fig. 2B). While all platforms reached saturation of known junctions, examination of the novel junctions showed that unlike Illumina and ONT Direct RNA, the ONT cDNA platform did not reach saturation. Novel junction reads can reflect the ability to capture novel transcripts, or alternatively, it can be indicative of junction mapping inaccuracies due to sequencing errors (ONT cDNA exhibited the highest error rate, see Table 1). To gain further insight, we partitioned between junctions that were detected by a single read and those with at least two reads, i.e., with the assumption 
that the abundancy of the junction made them more reliable. ONT cDNA had more unannotated junctions (67\%) compared to the other platforms (Fig. 2C), yet most of these novel and partial junctions were detected with a single read (52\%) and are therefore less trustworthy. Analysis of read distribution over exonic features (Fig. 2D) showed that the proportion of coding DNA sequence (CDS) exons was the highest in Illumina TruSeq (0.63), and that the $5^{\prime}$ and $3^{\prime}$ UTR exons were less represented in Illumina TruSeq in comparison to the ONT reads ( 0.35 vs at least 0.55 in ONT). The representation of introns and intergenic regions adjacent to annotated genes comprised a small fraction $(<0.03)$ of the reads, yet the ONT platform had a higher representation (Supplementary Fig. 1D). In accordance with this observation, read coverage on the gene body (Fig. 2E) showed that Illumina TruSeq's coverage was significantly reduced at the $5^{\prime}$ and $3^{\prime}$ ends of the gene (p-values of 0.001 and 0.00025 for the $10 \%$ and $90 \%$ gene body percentiles, respectively, see Methods). The reduced coverage of Illumina TruSeq at the $3^{\prime}$ of the gene body in comparison to ONT sequencing was also demonstrated previously [24,28]. This bias can be a result of the sequencing protocol, in which transcripts are sequenced from the $3^{\prime}$ to the $5^{\prime}$ end in the ONT Direct RNA, and can be truncated due to fragmentation during the library preparation, or pore blocking during sequencing. Such 3' bias has been shown also for ONT CDNA for the same reasons. Gene RPKM saturation analysis revealed a similar relative error rate per quantile of gene expression levels, upon subsampling in all three platforms (Supplementary Fig. 1E). The above-described quality features observed for Day4 samples were similar when analysing the Undiff datasets (Supplementary Fig. 1). The major difference between the platforms described here could possibly account for some of the differences in the analyses results to be described in this study.

\section{Comparison of gene expression levels}

We next performed a gene level analysis and observed on average, $0.6 \mathrm{M}, 1.6 \mathrm{M}$ and $36.9 \mathrm{M}$ reads mapped to genes of the ONT Direct RNA, ONT cDNA and TruSeq Illumina datasets, respectively (Table 1), in accordance to the raw sequencing yields. The biological replicate samples from the same platform were grouped together and exhibited high similarity (Fig. 3A, 3B), with a Spearman correlation coefficient in the range of 0.92-0.99. Furthermore, datasets collected at the same differentiation state from different platforms also exhibited a high correlation coefficient value, which ranged between 0.84-0.91 (Fig. 3A). In contrast, between different differentiation state samples (Undiff and Day4), and different platforms, a minimum correlation coefficient of 0.75 was reached. Similarly, principal component analysis (PCA) found the differentiation state to be the main source of variation, with PC1 explaining $69 \%$ of the variation, whereas $\mathrm{PC} 2$, which depicts the difference between the sequencing technologies, explaining $18 \%$ of the variation (Fig. $3 \mathrm{~B}$ ).

\section{Detection of differentially expressed genes across differentiation states}

Statistically significant differentially expressed genes (DEGs) between Day4 and Undiff were separately detected for each dataset (see methods). In total, 1,834, 2,058 and 8,168 DEGs were detected using ONT Direct RNA, ONT cDNA and Illumina TruSeq, respectively, indicating that the cells underwent numerous significant changes in the transition between the Undiff and Day4 states (Fig. 3C). As expected, the 
number of DEGs detected in the ONT datasets was low, due to fewer reads and consequently, lower detected gene expression levels (see difference in x-axis scale in Supplementary Fig. 2). We detected 1,112 DEGs overlapping in both ONT cDNA and ONT Direct RNA datasets, and most of them $(1,034)$ were also detected in Illumina TruSeq. Only three DEGs showed opposite directions among the three platforms (Supplementary Table 2).

The three DEG sets, comparing Day4 vs Undiff, also shared similar enriched canonical pathways (Ingenuity pathway analysis, Fig. 3D), among them, the following expected canonical pathways: the role of Oct4 in mammalian embryonic stem cell pluripotency and embryonic stem cell pluripotency in both human and mouse. Within the latter pathway, the genes Fzd1, Id3, Bmpr2 were upregulated and Nanog, Pou5f1, Zfp42, were downregulated (Fig. 3E).

\section{Isoform-based reconstruction and quantification of transcripts}

To address our goal to detect global changes in DS during ESC differentiation, and to leverage our long read datasets, we assembled and quantified transcripts using two reference-guided methods, namely StringTie2 and TALON. Initially, TALON was utilized to assemble transcript models from the aligned long reads, namely Day4 and Undiff, from both ONT cDNA and Direct RNA (analysis was performed with pooled replicates, only including transcripts that had at least five reads in any of the pooled samples, see methods). TALON identified and quantified 97,903 distinct transcript models, of them 28,575 were novel or partially novel (Supplementary Fig. 3A). The proportions of known transcripts detected by ONT Direct (62\%) was higher than ONT cDNA (44\%). A Spearman correlation coefficient of 0.55 was observed among the ONT CDNA pooled datasets (Day4 and Undiff) and 0.82 among the ONT Direct RNA pools (Supplementary Fig. 3B). There was an unexpectedly negligible similarity between the platforms (ONT cDNA and Direct RNA). We also quantified TALON transcripts using the twelve genome-aligned datasets (RNA-Seq mappings) with StringTie2 [23], to evaluate the similarity between biological replicated pairs including Illumina TruSeq datasets. Spearman correlation coefficients among the ONT replicates were in the range $0.68-0.69$, and 0.84 for the Illumina replicates (Supplementary Fig. $3 \mathrm{C}$ ). Similarities between the ONT and Illumina platforms were negligible.

As a second approach, the StringTie2 assembly was utilized on ONT and Illumina genome-aligned reads. A total of 147,769 transcripts (of them 16,852 novel) and 53,592 genes were assembled and quantified (see methods and Table 1). As in the TALON transcript analysis, the correlation of transcript expression among the biological replicates within the same platform was the highest for Illumina TruSeq samples (0.86), compared with ONT Direct RNA and ONT cDNA (0.63-0.66 respectively) (Supplementary Fig. 4A). Furthermore, the correlation coefficient between platforms was as low as 0.3 , implying dramatic differences in quantification across the platforms. A recent study has demonstrated that standard RNAseq is able to robustly recapitulate only about $50 \%$ of isoforms detected by long-read Iso-Seq sequencing [44]. The lack of quantified transcript similarities between the platforms in our study and, more alarmingly, the moderate similarity between the biological replicates tested in each platform, implies 
that the quantified levels obscure the real signal, and we therefore are not describing here an isoformbased analysis, aimed to detect DS.

\section{Detecting differential exon usages (DEUs)}

Towards our goal to detect DS we applied an exon count-based strategy, in which exon expression levels were quantified for all the datasets and compared between the differentiation states (see methods). The average total number of exons detected was $155 \mathrm{~K}$ for ONT and $261 \mathrm{~K}$ for Illumina (Table 1 ). This was in agreement with the high number of Illumina reads (10-100-fold more reads), and the longer length of the ONT reads (5-10-fold longer). The highest similarity of exon expression values was observed between the same platform biological replicates (Spearman correlation coefficient of at least 0.82, Fig. 4A). The Spearman correlation coefficient was at least 0.76 among the samples derived from the same sequencing platform, even if they originated from different differentiation states. Yet, the correlation coefficient between platforms was lower (range 0.55-0.82), and, interestingly, ONT Direct RNA was more similar to the Illumina TruSeq dataset.

The moderate similarity in exon expression between the platforms indicates a high variance of exon detected expression between the platforms. Comparison of DEUs, i.e., changes in the relative usage of exons between the datasets, was performed using DEXSeq [45]. This is a statistical generalized linear model with the following concept: for each exon (or part of an exon) and each sample, the tool counts the number of reads that map to this exon as well as how many reads map to any of the other exons of the same gene. The ratio of these two counts, and how it changes across conditions (in this case sequencing platforms or differentiation states), infers changes in the relative exon usage (see Methods). To identify DEUs that result from a technical issue, i.e., a consequence of the sequencing platform, we ran DEXSeq on datasets collected from the same differentiation state, yet sequenced by different platforms. Running this analysis is problematic due to the significant difference in read counts between the different platforms, therefore yielding thousands of DEUs. For example, comparing the ONT Direct RNA Day4 samples to Day4 samples acquired using the other platforms, resulted in 30,423 significant DEUs. Some of these exons were visually inspected using a genome browser. One convincing example was the protein tyrosine phosphatase 4A1 (Ptp4a1) gene (Supplementary Fig. 5), to which the platforms diverged in the coverage at both ends of the gene as well as in internal exons.

Due to the moderate similarity in exon expression between the platforms, differentiation state DEUs were sought for each platform separately. The number of exons utilized (passed the threshold, described in methods section) was $167,274,46,638$ and 46,418 for the Illumina TruSeq, ONT cDNA and ONT Direct RNA platforms, respectively. Overall, 7,534 significant DEUs were detected in 3,597 genes (Fig. 4B,Supplementary Table 3), with 237 DEUs being detected in more than one platform and only 27 DEUs being detected in all three sequencing platforms. Most of the DEUs were detected only by the lllumina TruSeq dataset $(6,836,91 \%)$. Furthermore, most of the DEUs detected by ONT were not detected by TruSeq $(69.1 \%, 483$ out of 698$)$. Analysis of DEUs that passed the filtrating criteria (50 counts in a least one sample) in all platforms, reveals a smaller proportion of DEUs detected only by TruSeq (Fig. 4C; 613, 
$72 \%)$. This difference between the platforms can be explained by a difference in both the specific exon coverage as well as the expression of all the other exons in the gene. The high DEU detection rate of Illumina TruSeq, is most likely due to its higher read coverage. However, this cannot be the only reason, since there are many genes which also had high coverage in ONT sequencing platforms, yet the relevant DEU was not detected. For example, the Psmc5 gene had a high coverage (thousands of reads) in ONT datasets (Supplementary Fig. 6), yet the specific DEU exon in the gene detected by Illumina TruSeq was barely expressed in any of the ONT samples.

Of all DEUs detected, 3,072 exons contained coding sequence (CDS) regions (Supplementary Table 3); 1,970 were in 'intron' exons (exhibiting an alternative donor/acceptor site within an intron, intron retention or exon skipping, and named herein as an intron), 2,054 were in 5' UTRs and 520 were in 3' UTRs. Interestingly, an analysis of the proportions of DEUs by exon category and platform indicated a decrease in detecting DEUs in 3' UTRs in TruSeq relative to their percentage in all exons (Fig. 4D, decrease for DEUs detected only by TruSeq from $90.7 \%$ to $83.5 \%$ ). This was in agreement with the read coverage proportions shown in Fig. 2D and 2E and to the positive bias of ONT reads to the $3^{\prime}$ end of transcripts.

Exploring the DEU length distributions per sequencing platform, revealed a significant difference between the TruSeq and the ONT platforms (Fig. 4E). For instance, the expressed exons' median lengths were 190, 120 and 103 bases for TruSeq, ONT cDNA and Direct respectively. This difference may be attributed to both the differences in read length and the assumptions underlying the mapping algorithms (i.e., STAR and minimap2).

To evaluate the relation between DEUs and DEGs, and their direction of regulation, we calculated the proportions of the DEUs to DEGs, categorized by the differential gene expression analysis information: same statistically significant trend ("same", both upregulated or downregulated); opposite trend, ("opposite"); or significantly unchanged ("unchanged") (Fig. 4F). We detected that among the three platforms, at most, only $37 \%$ of DEUs showed the same statistically significant trend as in the gene-level analysis. This suggests that the bulk of exon-based analysis revealed additional differential transcriptome changes which were not apparent at the gene level analysis.

Some of the genes with DEUs were already reported to exhibit DS events during mouse embryonic development. For example, Dnmt3b (DEUs found by ONT Direct RNA and Illumina TruSeq), Clk1 (DEUs identified by Illumina TruSeq and ONT cDNA) and Ctage5 (identified only by TruSeq) were found to exhibit DS in the transitional stage (from E8.0 to E9.0) [46]. Some of the genes detected only by Illumina TruSeq to have DEUs were previously reported, e.g., transcriptional initiation of a short Stra6 isoform (Stra6S) was found in mouse ESCs in response to RA [47] and novel alternative splicing variants of Klf4 were first identified in mouse ESCs [48]. Smarcb1 was found to undergo DS in ESCs as compared to Ebs [49] and in our analysis in ONT Direct RNA and ONT cDNA (and was PCR validated, see next section).

\section{Selection and validation of DEUs by qRT-PCR and RT-PCR}


Initially, we explored the correlation between the calculated DEXSeq exon expression-fold changes (Day4 vs Undiff) in the three platform, and their observed qRT-PCR-fold change values. Towards this aim, qRTPCR was performed on 42 exons, 26 of them DEUs from 19 different genes (Acot7, Aplp1, Ash2l, Caprin1, Egfl7, Gemin7, Hmgxb4, Mbd3, Mta1, Myl6, Nfu1, Pcolce, Rpl31, Rps24, Serf2, Tmsb10, Usp7, Wbp1, Zmynd8, see Supplementary Table 3), and 16 constitutive exons (i.e., exons which did not exhibit differential usage) from the same genes (Supplementary Table 4). The observed qRT-PCR-fold change values were highly correlated (Spearman correlation coefficient of at least 0.92) to their calculated DEXSeq-fold changes (Fig. 5A). Furthermore, a high correlation of at least 0.88 , was observed among the calculated DEXSeq fold changes (Supplementary Table 4).

For qRT-PCR validation of the DEXSeq predicted DEUs, 24 DEUs were selected from 17 different genes (a subset of the above). The selected DEUs were predicted to be significant by either one or more of the platforms: 14 by ONT cDNA, 13 by ONT Direct RNA and 18 by Illumina TruSeq. Some of the DEUs (13) were in the coding sequence and protein motifs (4 exons), thus presumably affecting the protein function (Supplementary Table 3), and some were in 'introns' (14). To validate the DEUs, we selected more than one exon per gene, either a DEU or a constitutive exon or at least two DEUs. The experimental qRT-PCR mean expression values of DEUs from 11 genes along with their calculated DEXSeq values are presented in Fig. 5B, Fig. 6C and Fig. 7C. qRT-PCR demonstrated that these DEUs significantly changed between the days, while the additional exon from the same gene showed an opposite trend or an insignificant change. For instance, the gene Hmgxb4 had a significantly high expression of the DEU named Hmgxb4_TruSeq_74998835 (detected as a significant DEUs in TruSeq) in the qRT-PCR Undiff samples, whereas the additional DEU named Hmgxb4_direct_75016222 (detected as a significant DEU in TruSeq and Direct) had a significantly high expression in qRT-PCR Day4 samples. In other genes (Egfl7, Nfu1, Usp7, Wbp1 and Mta1) both the DEU and the constitutive exon were upregulated in the same differentiation state but not to the same extent, as evident by their log2 fold-change of Day4 vs Undiff by both DEXSeq and qRT-PCR (Supplementary Fig. S7 and Supplementary Table 4). For the gene Rps24 the exon expression values demonstrated were in the trend expected, yet in order to better validate the DEU, a different constitutive exon should have been selected (Supplementary Fig. S7 and Supplementary Table 4).

As a second validation approach, we performed RT-PCR to confirm DEU events of alternative splicing in 'intronic' coding exons within the genes: Zfp207, Mark3, Smarcb1, Mta1 and Enah, detected by ONT Direct (two DEUs were detected also by TruSeq and two others also by ONT cDNA). RT-PCR was performed using primer pairs designed to target the immediately flanking constitutive exons, and detected the two expected amplified product sizes, in accordance to the differentiation day (Fig. 5C, Supplementary Table 5B). The presence of additional bands can be explained by additional isoforms or non-specific amplification. To summarize, we validated DEUs from 20 genes by qRT-PCR and RT-PCR, predicted by DEXSeq analysis in one or more of the sequencing platforms.

\section{Exploring DEUs in the Aplp1 gene}


An example of a gene that exhibited DEUs was the amyloid-like protein 1 (Aplp1) gene, a member of a family of proteins involved in neuronal development and in dementia [50]. This gene was also identified as a DEG only by ONT cDNA. Overall, we identified 12 DEUs within this gene, many of them are in the $5^{\prime}$ end of the gene and were identified mostly by ONT Direct in a region of the gene that contains the "E1" domain (Fig. 6A and Supplementary Table 3). The Aplp1 gene harbours transcripts (for example: ENSMUST00000207514.1 and ENSMUST00000208404.1) that might not encode a protein, and presumably have alternative transcription start sites (TSS) that are shorter than the protein coding transcript. We identified two internal DEUs (Aplp1_cDNA_30436895 and Aplp1_cDNA_30440303, Fig. 6B) that were significantly upregulated in the Undiff state, as detected by the TruSeq and ONT cDNA platforms. Some of the ONT reads covering and starting at these DEUs are in agreement with the start of the short transcripts. These DEUs were validated by qRT-PCR and their expression was demonstrated to be significantly different between Undiff and Day4, as expected (Fig. 6C and Supplementary Table 4).

In an attempt to decipher differentially expressed transcript models containing and starting at the two validated DEUs, we explored the StringTie2 assembled and quantified transcripts (Supplementary Fig. 4B). The analysis revealed two short transcripts that were more highly expressed in the Undiff state within the ONT cDNA datasets. Similarly, TALON assembled two short transcripts with a TSS similar to ENSMUST00000208404.1, starting with the DEU Aplp1_cDNA_30440303, which were more abundant in Undiff. Yet, no evidence was found for a transcript starting at the DEU Aplp1_cDNA_30436895 (data not shown).

\section{Exploring DEUs in the Mbd3 gene}

Gene-level analysis did not identify the Mbd3 (methyl-CpG binding domain protein 3) gene as a DEG between Undiff and Day4 samples, yet exon-based analysis identified DEUs within this gene. Mbd3 is an essential pluripotency gene, and is a key component of the NuRD chromatin remodelling complex [51, 52]. Four Mbd3 gene DEUs were detected by either one or more platforms (in GENCODE, these are three exons. However, in the analysis, one exon was split into two since there were transcripts in which this exon was only partially overlapping) (Fig. 7 and Supplementary Fig. 8). Two of the exons were upregulated in Undiff (Mbd3_cDNA_80395202 and Mbd3_cDNA_80395286 are in fact part of the same exon), and two were upregulated in Day4 samples (Mbd3_cDNA_80395436 and Mbd3_cDNA_80399218, Fig. 7A and 7B). These exons, along with a constitutive exon were selected for validation by qRT-PCR (Fig. 7C), and their expression was demonstrated to be significantly different between Undiff and Day4, as expected.

ONT reads revealed an annotated transcript start of a Mbd3 short isoform, presumably upstream to the DEUs upregulated in Undiff (Mbd3_cDNA_80395202 and Mbd3_cDNA_80395286) (Fig. 7D and Supplementary Fig. 9). Spliced isoforms for Mbd3 are reported in the sequence databases, and result in different protein products (Fig. 7E). Specifically, the N-terminal methyl-CpG binding and MBD2/MB3_p55 binding domains are shorter in the known transcripts (i.e., ENSMUST00000105347.1, Fig. 7E), or entirely missing in ENSMUST00000125618.1 (annotated as non-coding). Yet, the above annotated isoforms did 
not initiate at the novel Mbd3 TSS as detected here. Supporting the above described TSS, is a reported promoter (located at chr10:80395362-80395421), in addition to the canonical promoter (EPDnew UCSC track in Fig. 7D), that suggests the presence of an additional mechanism for transcriptional regulation. A second indication for the above TSS is that its $5^{\prime}$ exon encodes an ORF in frame with the Mbd3 gene, extending by 49 amino acids an internal exon encoding the MBD2/MB3_p55 binding domain (Supplementary File 1). Part of this ORF (28 amino acids) overlaps an exon in ENSMUSP00000100985.1. An alignment generated by TBLASTX with the $5^{\prime}$ end of our predicted shorter transcript, showed that the 49 amino acids were conserved in other species, such as Rattus norvegicus (GenBank: EDL89285.1) (Fig. 7F).

Even though this isoform does not exist in the public sequence repositories, experimental support for the functionality of this alternative transcript was reported by Eu et al. [53]. They demonstrated mouse ESCs expression of an $\mathrm{Mbd} 3$ isoform ( $\mathrm{Mbd} 3 \mathrm{C}$ ) bearing a unique 50 -amino-acid $\mathrm{N}$-terminal region that is necessary for interaction with the histone $\mathrm{H} 3$ binding protein WDR5. This interaction creates a unique NuRD complex variant that specifically functions in ESCs.

We further explored the presence of the novel short Mbd3 and its differential upregulation in the Undiff state, within our transcript assembly datasets. No Mbd3 novel transcript was detected in the StringTie2 assembly. Furthermore, transcript expression plots for the Mbd3 gene did not reveal a coherent transcript expression pattern between the replicates or between the platforms (using StringTie2 FPKM quantification, Supplementary Fig. 4C). Therefore, StringTie2 did not support transcript models explaining the validated DEUs. In contrast, in TALON-assembled transcripts predicting for the Mbd3, we identified five known isoforms and seven novel transcripts with at least five supporting reads (Supplementary Fig. 10). One of the detected novel transcripts (TALONT000325042), initiated downstream to the alternative TSS described above, was 10-fold more abundant in Undiff in both ONT platforms (Fig. 7B and 7E, Supplementary Table 6).

\section{Discussion}

This work aimed to detect transcriptome changes in the gene, transcript and exon levels using three sequencing platforms, namely Illumina short reads, ONT cDNA and ONT Direct long reads, towards the discovery of DS events in mouse ESCs undergoing differentiation.

At the gene level, the differentiation state was the dominant cause for variations in gene expression values. While there was a high overlap in the DEGs detected by the sequencing platforms and all DEG lists were enriched for embryonic stem cell pluripotency pathways, the Illumina technology had an advantage in detecting more DEGs due to the higher number of reads and thus higher transcriptome coverage.

At the isoform-based analysis, the agreement in expression levels between platforms decreased. Therefore, comparing transcripts assembled and quantified (using TALON and StringTie2) across the platforms resulted in a weak or non-detectable correlation. Despite the premise of long reads in 
constructing transcripts, their low throughput and low sequence accuracy demonstrate that generating accurate transcriptomes from imperfect RNA reads is still a challenge. This was also seen in other studies [22, 32]. Moreover, it is hard to distinguish whether reads with premature starts and ends indicate native internal transcription start or end sites, or technical issues such as fragmented reads or blocked pores. This phenomenon inflates the number of reconstructed transcripts. Nevertheless, they can also be genuine alternative TSSs, as we demonstrated for the Mbd3 gene that are upregulated in the Undiff state, and encode a shorter Mbd3 protein that lacks the N-terminal methyl-CpG DNA binding domain.

We have shown that each of the technologies presents various sequence biases, which is a consequence of differences in reads length, number of reads, error rates, biased coverage along the gene body and the ability to accurately detect exon junctions. These biases can explain the low reproducibility of reconstructed transcripts quantification. A recent hybrid approach using long sequences and additional quality control and validation steps using the short reads was used to improve the final reconstructed transcripts set [28]. However, sequencing biases that we observed, i.e., decreased coverage of the short reads at the transcript UTRs, still need to be addressed in the hybrid approach. In addition, the short reads cannot aid in identifying true internal transcription start or end sites. Ongoing advances in technology and computational algorithms that increase the accuracy of sequence and isoforms detection, such as Sahlin et al. [21], will further enhance the potential of long-read RNA sequencing.

The exon-based strategy enabled the detection of numerous statistically significant DEUs, by both short and long sequencing platforms. Interestingly, most of DEUs were detected uniquely by one of the platforms: the long reads exhibited an advantage in detecting DEUs at the $3^{\prime}$ UTRs, whereas short reads had an advantage in detecting an order of magnitude more DEUs than with the ONT platforms. The fact that most of the DEUs were not found in genes detected as DEGs, suggests that numerous DS events occur during ESC differentiation, and could not be otherwise detected at the gene-level analysis. Only a fraction of DEUs were detected by all platforms (27 out of 7,534), still, DEUs from 20 genes (detected by one or more platforms) were validated by qRT-PCR and RT-PCR, including DEUs that are protein coding and alternatively spliced introns in key genes of the ESCs differentiation process (Mbd3 and Aplp1), or in chromatin modification (Ash2I, Mbd3, Mta1, Smarcb1, Usp7) as well as others. Taken together, these observations pose a promising means of deciphering DS events, and highlight that sequencing platforms reveal complementary information.

This work provides an extensive repository of short and long reads, along with gene- isoform- and exonbased analyses, profiling the transcriptomic changes upon RA-induced mouse ESC differentiation, which can be used as a resource for discovering functional diversity, and to facilitate the development of new analysis methods by the scientific community.

\section{Conclusions}

In this study, we explored three sequencing platforms, namely Illumina short reads, ONT cDNA and ONT Direct long reads, and found that at the gene-level expression, Illumina short reads identified more 
changes due to its higher coverage, yet the three platforms discovered similar transcriptomic profiles. In an attempt to discover DS during mouse ESC differentiation, quantification of reconstructed transcripts was found to be irreproducible. Thus, even with the use of long reads, precise transcript structure reconstruction and transcript quantification remain challenging. Nevertheless, we demonstrated that exon-based detection of DUEs, developed for short reads, was also applicable with long reads, and that the three sequencing platforms complement one another and are reliable as validated by PCR.

\section{Methods}

\section{Cells and RNA extraction}

R1 mouse embryonic stem ( $m E S$ ) cells were maintained in mES growth medium (DMEM, fetal bovine serum (FBS), L-glutamine, non-essential amino acids, penicillin/streptomycin, beta-mercaptoethanol and Leukaemia inhibitory factor), and named herein as sample Undiff. Embryoid bodies (Ebs) were generated from R1 single-cell suspensions ( 35000 cells $/ \mathrm{ml}$ ) in $\mathrm{mES}$ growth medium without Leukaemia inhibitory factor in low adherence dishes and grown for four days. Thereafter, they were treated for four days with $2 \mu \mathrm{M}$ RA (Sigma R2625), and termed sample Day4. RNA was extracted using the RNeasy Mini Kit (Qiagen), and its quality was assessed using TapeStation (Agilent). Poly(A) RNA was isolated from the total RNA using the Dynabeads mRNA DIRECT kit (ThermoFisher Scientific) according to the manufacture's protocol.

Illumina library preparation and sequencing

A total of $1 \mu \mathrm{g}$ RNA was processed using the Illumina TruSeq RNA Sample Preparation Kit v2 protocol. Libraries were evaluated by Qubit and TapeStation. Sequencing libraries were constructed with barcodes to allow multiplexing. Between 39 and 59 million paired-end reads were sequenced ( $2 \times 101$ bases) per sample (Table 1), on Illumina HiSeq Rapid 2500 instrument using protocols RTA (1.17.21.3) and HCS (2.0.12.0).

ONT library preparation and sequencing

For cDNA-PCR library preparation, a total of $50 \mathrm{ng}$ poly(A) RNA was used as input. Libraries were prepared according to manufacturer's protocols using the CDNA-PCR Sequencing Kit (SQK-PCS108, ONT, Oxford, United Kingdom; 1D - meaning that the template and the complement strands are sequenced as individual strands). Input of $500 \mathrm{ng}$ poly(A) RNA was used for the Direct RNA library preparation kit (SQKRNA002, ONT, Oxford, United Kingdom). Both types of libraries were sequenced using the ONT MinION 106D R9 version flow cells. MinKNOW software (version 3.1.8, ONT) was used to run each flow cell for 48 h.

Quantitative real-time reverse transcription polymerase chain reaction (qRT-PCR)

Complementary DNA was synthesized from $500 \mathrm{ng}$ total RNA using the PrimeScript RT Reagent Kit (TAKARA) according to the manufacturer's instructions, with both oligo-dT primers and random 
hexamers. qRT-PCR primers were designed for DEUs and constitutive exons (for the genes Serf2 and Tmsb10, only one DEU was designed and no constitutive exon was selected) using either primer3 (http://bioinfo.ut.ee/primer3-0.4.0/) or Blast-Primer (https://www.ncbi.nlm.nih.gov/tools/primer-blast/) and are listed in Supplementary Table 5A. qRT-PCR analyses were performed with SYBR Green (Applied Biosystems, Foster City, CA, USA) on five replicates per exon and differentiation day. Signals (CT) were normalized to genes and exons that were found to have high and similar expression levels in all platforms and differentiation days (Pum2, Sec24d, Copg1). To compare between Day4 and Undiff, we performed a t-test on $2^{\wedge}(-\Delta C T)$ of Day4 vs Undiff, and $p$-values were corrected with a Benjamini-Hochberg adjustment. Fold-changes were calculated between mean Day4 values $2^{\wedge}(-\Delta \mathrm{C} T)$ and mean Undiff values.

Reverse transcription polymerase chain reaction (RT-PCR)

RT-PCR primers were designed from constitutive exons that flank DEUs classified as introns and yield an amplified product $<500 \mathrm{bp}$ (primers are listed in Supplementary Table 5B). The Copg 1 gene was used as a loading control. cDNA was prepared as described above, and PCR was done using the primers and KAPA Hifi HotStart ReadyMix (Roche; Cape Town, South Africa). The cycling acquisition program used was: initial denaturation at $98^{\circ} \mathrm{C}$ for $2 \mathrm{~min}$, followed by 35 cycles of denaturation temperature at $98^{\circ} \mathrm{C}$ for $20 \mathrm{sec}$, annealing at $64^{\circ} \mathrm{C}$ for $30 \mathrm{sec}$ and elongation at $72^{\circ} \mathrm{C}$ for $30 \mathrm{sec}$; and a final elongation step at $72^{\circ} \mathrm{C}$ for 1 min. RT-PCR products, assayed in triplicates per differentiation state, were resolved using $2 \%$ SeaKem LE (Lonza; Rockland, USA) agarose gel.

Genome browsers

The UCSC genome browser [54] (https://genome.ucsc.edu/s/bareket/mm10\%2DES\%2Dtranscriptome\%2Danalysis) and IGV [55] were used to visualize the reads and the assembled transcripts on selected genomic regions. Searching for protein sequence similarities with novel Mbd3 transcript start was performed by running TBLASTX against the nt database at NCBI (https://blast.ncbi.nlm.nih.gov/).

Bioinformatics analysis of Illumina sequences

Raw reads were analysed using the UTAP transcriptome analysis pipeline [56]. Initially, reads were trimmed using cutadapt [57] to remove TruSeq adaptors, with the parameters: -times 2 -q $20-\mathrm{m} 25$. Reads were mapped to the genome (mm10, GENCODE annotation) using STAR (v2.4.2a) [58], with the following parameters: -alignEndsType EndToEnd, -outFilterMismatchNoverLmax 0.05, -twopassMode Basic.

Bioinformatics analysis of ONT sequences

Direct RNA reads were acquired using the MinION software from Oxford Nanopore Technologies (ONT), and base-called using either ONT albacore (MinKNOW) or Guppy software (Supplementary Table 1). Raw reads were converted from fast 5 to fastq format and processed to base calls using Poreplex (https://github.com/hyeshik/poreplex), trimmed to remove any 3' adapter sequences, and filtered to 
remove chimeras (unsplit reads fused of two or more RNAs), with the parameters -trim-adapter --basecall -filter-chimera.

ONT cDNA raw reads from the "skip" folder were base-called using Guppy (--flowcell FLO-MIN106 -kit SQK-PCS108). These reads were merged with reads from the pass folders, and processed using porechop (v0.2.3) to remove adaptors.

The pre-processed reads were aligned to the Mus musculus genome (mm10, UCSC) using minimap2 ( $v$ 2.10) adjusted for long-read spliced alignment (-x splice, --secondary = no, --MD). SAM alignment files were sorted, and converted to indexed BAM files. For DEXSeq analysis, the resulting primary aligned reads were marked with "NH:i:1" tags using the UNIX awk command. The percent of reads multiple aligned was below $2.4 \%$.

Gene-level analysis

Reads on genes were counted using htseq-count [59] with mm10 annotation (downloaded from iGenomes UCSC), and considering the strandedness of the samples (Direct RNA samples were run as strand-specific; -s yes).

Spearman correlation coefficient analysis was performed on the raw counts using the cor function in the $\mathrm{R}$ stat package [60] and heatmaps were created using the gplots package (heatmap.2). PCA analysis was performed on log-normalized values, computed with DESeq2 (rlog function, blind = TRUE) [61] using the R prcomp package.

Differential gene expression analysis was performed separately on the count matrix for each of the platforms, using the UTAP pipeline [56]. Specifically, normalization of the counts and differential expression analysis were performed using DESeq2 with the parameters: betaPrior $=$ True, cooksCutoff $=$ FALSE, independentFiltering $=$ FALSE. The following criteria were used to select differentially expressed genes (DEG): adjusted P-value $<=0.05, \| \log 2$ FoldChange| $>=1$ and baseMean $>=5$.

Enrichment analysis of canonical pathways of the three DEG lists along with their log-fold change values (Day4 vs Undiff) was performed using Ingenuity Pathway Analysis (QIAGEN Inc., https://www.qiagenbioinformatics.com/products/ingenuitypathway-analysis). The DEG lists enrichment results were compared using the 'comparison' feature in IPA.

Comparison of sequence quality control features

Various sequence quality control features were extracted from the aligned reads (separate BAM file for each sample and platform) using the RSeQC tool v 2.6.4 [62] and NanoPlot v 1.14.1 [63]. The features extracted were GC content per read (read_GC.py), read distribution over genome regions (read_distribution.py), junction novelty (junction_annotation.py), junction saturation (junction_saturation.py), genebody coverage (genome_coverage.py) and RPKM saturation (rpkm_saturation.py, ONT Direct RNA was run with $-d$ ',$+++-'$ ). The read coverage analysis on gene body 
was done by scaling all transcripts to 100 bases and calculating the proportions of reads covering each nucleotide position. Statistical significance was calculated for specific gene body percentile coverage $(10 \%, 90 \%)$ using ANOVA. The RSeQC output files per sample were merged using R and Excel to a single plot, and in some of the plots, the replicate values were averaged before plotting. GC content of the transcriptome was calculated using bedtools nuc on all transcripts [64]. Alignment error rates were calculated using NanoPlot.

Exon-count based analysis

The GENCODE annotation gtf file vM15 was processed by running the python script dexseq_prepare_annotation.py (option $-r$ no). The exons were quantified with dexseq_count.py using options:" -r pos -s no", except for ONT Direct RNA that ran with the option "-s reverse", and "-p yes" for Illumina TruSeq. Spearman correlation coefficient analysis of the raw counts (merged using DESeq2 DESeqDataSetFromHTSeqCount) was as described above for the gene level analysis.

Differential exon usages (DEUs) between Undiff and Day4 (or the day attribute) samples was detected using R 3.5.1 and DEXSeq R package (v1.26.0) [45], run separately for each platform. Initial filtering was performed to keep exons that have 50 counts in a least one sample, and at least 10 reads in "other exons" in all samples. Differentially used exons were found using the DEXSeq function testForDEU using the full model (fullModel $=$ sample + exon + day:exon), and a reduced model (reducedModel $=\sim$ day + exon). Analysis of exons that are differentially expressed between the sequencing platforms for a certain differentiation state (either Undiff or Day4), was performed by including the platform category in the model (instead of the day). Criteria for selecting differentially expressed exons were adjusted P-value $<=$ 0.05 and an absolute |log2FoldChange| >=1, and a minimal exon length of 18 bases. Annotation of the DEUs (between Undiff and Day4) into 5' UTR, 3' UTR, CDS or intron (meaning alternatively spliced intron) categories was done by running bedtools intersect (parameters $-\mathrm{s}-\mathrm{u}$ ) using a bed file of the DEUs and exon category-derived GTF files. These GTF files were derived using the R package GenomicFeatures [65] and GENCODE (vM15) annotation. The outputs were intersected with the original GENCODE GTF to add the gene_id using bedtools [64] (parameters: intersect -s -wa - $f$ 1.0). Annotation of protein domains was done by intersection with UCSC table unipDomain (release 2020_06).

Quantifying and characterizing isoform-level expression of genes with TALON

The TALON package [22] was applied to identify and quantify isoforms in ONT samples (cDNA and Direct RNA). The alignments, pooled from both replicates, were pre-processed with talon_label_reads to remove artifacts of internal priming with A-rich sequences ( $20 \mathrm{bp}$ window). The TALON database was initialized from the GENCODE (vM15) annotation with talon_initialize_database module (parameters: - $10-5 p 500$ -3p 300). The TALON module was applied for transcript annotation (parameters: -cov 0.9 -identity 0.8), keeping transcript models with $>5$ reads in at least one of the pooled replicates. Overall, each pool identified $<48.3 \mathrm{~K}$ distinct transcripts that were merged to a total of 97,904 distinct transcript models ("merged GTF") (see Supplementary Fig. 3A). A Spearman correlation was calculated between the transcript abundances (quantified using talon_abundance function) for the pooled replicates. In addition, 
the pooled transcripts ("merged GTF") were quantified for each sample (using the aligned reads), including the Illumina TruSeq genome-aligned sequences using StringTie2 (see details below for parameters). The FPKM values from the t_data.ctab file outputs were used to calculate Spearman correlation coefficients.

Transcript assembly and quantification with StringTie2

StringTie2 [23] version 2.1.4 was used to run guided assembly with the GENCODE (vM15) annotation (parameter $-G-B$ ) from the aligned reads. The parameter (-L ) was implemented for the ONT reads and the parameter (-rf) for the ONT Direct RNA reads. The transcripts were then merged to one gtf file (--merge) and estimates of transcript abundance were done by running StringTie2 with the addition of the parameter (-e). Overall, 147,769 distinct transcript models were identified. Spearman correlations were calculated on the transcripts FPKM counts. Expression plots were prepared with the R package ballgown v 2.22.0 [66] function plotTranscripts using FPKM measures.

Protein domain analysis

Protein domains in Mbd3 isoforms were inferred by DoChaP [67] and Pfam database searches [68].

\section{Abbreviations}

Differential exon usage (DEU)

Differential splicing (DS)

Differentially expressed gene (DEG)

Coding sequence (CDS)

Embryoid bodies (EBs)

Embryonic stem cells (ESCs)

Fetal bovine serum (FBS)

Mouse embryonic stem (mES)

Oxford Nanopore Technology (ONT)

Principle component analysis (PCA)

Quantitative Reverse Transcription PCR (qRT-PCR)

Retinoic acid (RA)

Reverse transcription PCR (RT-PCR)

Page 17/35 
Standard deviation (SD)

Transcription start site (TSS)

Untranslated region (UTR)

\section{Declarations}

\section{Ethics approval and consent to participate}

Not applicable

\section{Consent for publication}

Not applicable

\section{Availability of data and materials}

Custom tracks are available on the UCSC browser using the session:

https://genome.ucsc.edu/s/bareket/mm10\%2DES\%2Dtranscriptome\%2Danalysis. The data discussed in this publication have been deposited in NCBI's Gene Expression Omnibus and are accessible through GEO Series accession number GSE156371.

\section{Competing interests}

The authors declare that they have no competing interests

\section{Funding}

This work was supported by the Weizmann Institute of Science - Staff Scientists Internal Grant Program, granted to: DL and EA.

\section{Authors' contributions}

$\mathrm{DL}$ and $\mathrm{BD}$ were responsible for the bioinformatics analysis design, implementation and presentation. EA and YF were responsible for cell culture and RNA extraction. HKS, MK and DP were responsible for ONT library preparation and sequencing. MK was responsible for design and performing PCR analysis. MK, DL and BD performed PCR data analysis. DL and BD wrote the manuscript. All authors were responsible for the critical evaluation of the manuscript. All authors read and approved the final manuscript.

\section{Acknowledgements}

We thank Vitaly Golodnitsky for IT assistance, Daniela Amann Zalcenstein for sequencing and preparing the Illumina libraries, Ron Rotkopf for statistical advice and Yehudit Posen and Mechael Kanovsky for editing the manuscript. 


\section{References}

1. Marioni JC, Mason CE, Mane SM, Stephens M, Gilad Y: RNA-seq: an assessment of technical reproducibility and comparison with gene expression arrays. Genome Res 2008, 18(9):1509-1517.

2. McGettigan PA: Transcriptomics in the RNA-seq era. Curr Opin Chem Biol 2013, 17(1):4-11.

3. Pan Q, Shai O, Lee LJ, Frey BJ, Blencowe BJ: Deep surveying of alternative splicing complexity in the human transcriptome by high-throughput sequencing. Nat Genet 2008, 40(12):1413-1415.

4. Rosenkranz R, Borodina $\mathrm{T}$, Lehrach $\mathrm{H}$, Himmelbauer $\mathrm{H}$ : Characterizing the mouse ES cell transcriptome with Illumina sequencing. Genomics 2008, 92(4):187-194.

5. Consortium SM-I: A comprehensive assessment of RNA-seq accuracy, reproducibility and information content by the Sequencing Quality Control Consortium. Nat Biotechnol 2014, 32(9):903914.

6. Leshkowitz D, Feldmesser E, Friedlander G, Jona G, Ainbinder E, Parmet Y, Horn-Saban S: Using Synthetic Mouse Spike-In Transcripts to Evaluate RNA-Seq Analysis Tools. PLoS One 2016, 11(4):e0153782.

7. Kanitz A, Gypas F, Gruber AJ, Gruber AR, Martin G, Zavolan M: Comparative assessment of methods for the computational inference of transcript isoform abundance from RNA-seq data. Genome Biol 2015, 16:150.

8. Steijger T, Abril JF, Engström PG, Kokocinski F, Hubbard TJ, Guigó R, Harrow J, Bertone P, Consortium R: Assessment of transcript reconstruction methods for RNA-seq. Nat Methods 2013, 10(12):11771184.

9. Mehmood A, Laiho A, Venäläinen MS, McGlinchey AJ, Wang N, Elo LL: Systematic evaluation of differential splicing tools for RNA-seq studies. Brief Bioinform 2020, 21(6):2052-2065.

10. Weirather JL, de Cesare M, Wang Y, Piazza P, Sebastiano V, Wang XJ, Buck D, Au KF: Comprehensive comparison of Pacific Biosciences and Oxford Nanopore Technologies and their applications to transcriptome analysis. F1000Res 2017, 6:100.

11. Sessegolo C, Cruaud C, Da Silva C, Cologne A, Dubarry M, Derrien T, Lacroix V, Aury JM:

Transcriptome profiling of mouse samples using nanopore sequencing of cDNA and RNA molecules. Sci Rep 2019, 9(1):14908.

12. Garalde DR, Snell EA, Jachimowicz D, Sipos B, Lloyd JH, Bruce M, Pantic N, Admassu T, James P, Warland A et al: Highly parallel direct RNA sequencing on an array of nanopores. Nat Methods 2018, 15(3):201-206.

13. Byrne A, Beaudin AE, Olsen HE, Jain M, Cole C, Palmer T, DuBois RM, Forsberg EC, Akeson M, Vollmers C: Nanopore long-read RNAseq reveals widespread transcriptional variation among the surface receptors of individual B cells. Nat Commun 2017, 8:16027.

14. Ip CLC, Loose M, Tyson JR, de Cesare M, Brown BL, Jain M, Leggett RM, Eccles DA, Zalunin V, Urban $\mathrm{JM}$ et al: MinION Analysis and Reference Consortium: Phase 1 data release and analysis. F1000Res 2015, 4:1075. 
15. Soneson C, Yao Y, Bratus-Neuenschwander A, Patrignani A, Robinson MD, Hussain S: A comprehensive examination of Nanopore native RNA sequencing for characterization of complex transcriptomes. Nat Commun 2019, 10(1):3359.

16. Seki M, Katsumata E, Suzuki A, Sereewattanawoot S, Sakamoto Y, Mizushima-Sugano J, Sugano S, Kohno T, Frith MC, Tsuchihara K et al: Evaluation and application of RNA-Seq by MinION. DNA Res 2019, 26(1):55-65.

17. Li R, Ren X, Ding Q, Bi Y, Xie D, Zhao Z: Direct full-length RNA sequencing reveals unexpected transcriptome complexity during. Genome Res 2020, 30(2):287-298.

18. Parker MT, Knop K, Sherwood AV, Schurch NJ, Mackinnon K, Gould PD, Hall AJ, Barton GJ, Simpson GG: Nanopore direct RNA sequencing maps the complexity of Arabidopsis mRNA processing and $\mathrm{m}$. Elife 2020, 9.

19. Gleeson J, Lane TA, Harrison PJ, Haerty W, Clark MB: Nanopore direct RNA sequencing detects differential expression between human cell populations. BioRxiv 2020.

20. Workman RE, Tang AD, Tang PS, Jain M, Tyson JR, Razaghi R, Zuzarte PC, Gilpatrick T, Payne A, Quick $\mathrm{J}$ et al: Nanopore native RNA sequencing of a human poly(A) transcriptome. Nat Methods 2019, 16(12):1297-1305.

21. Sahlin K, Medvedev P: Error correction enables use of Oxford Nanopore technology for reference-free transcriptome analysis. Nat Commun 2021, 12(1):2.

22. Wyman D, Balderrama-Gutierrez G, Reese F, Jiang S, Rahmanian S, Forner S, Matheos D, Zeng W, Williams BA, Trout D: A technology-agnostic long-read analysis pipeline for transcriptome discovery and quantification. BioRxiv 2020:672931.

23. Kovaka S, Zimin AV, Pertea GM, Razaghi R, Salzberg SL, Pertea M: Transcriptome assembly from long-read RNA-seq alignments with StringTie2. Genome Biol 2019, 20(1):278.

24. Amarasinghe SL, Su S, Dong X, Zappia L, Ritchie ME, Gouil Q: Opportunities and challenges in longread sequencing data analysis. Genome Biol 2020, 21(1):30.

25. Cui J, Shen N, Lu Z, Xu G, Wang Y, Jin B: Analysis and comprehensive comparison of PacBio and nanopore-based RNA sequencing of the. Plant Methods 2020, 16:85.

26. Parker MT, Knop K, Sherwood AV, Schurch NJ, Mackinnon K, Gould PD, Hall AJ, Barton GJ, Simpson GG: Nanopore direct RNA sequencing maps the complexity of Arabidopsis mRNA processing and m6A modification. Elife 2020, 9.

27. Chen Y, Davidson N, Wan YK, Patel H, Yao F, Low HM, Hendra C, Watten L, Sim A, Sawyer C: A systematic benchmark of Nanopore long read RNA sequencing for transcript level analysis in human cell lines. bioRxiv 2021.

28. Wright DJ, Hall NAL, Irish N, Man AL, Glynn W, Mould A, Angeles AL, Angiolini E, Swarbreck D, Gharbi $\mathrm{K}$ et al: Long read sequencing reveals novel isoforms and insights into splicing regulation during cell state changes. BMC Genomics 2022, 23(1):42.

29. Uapinyoying P, Goecks J, Knoblach SM, Panchapakesan K, Bonnemann CG, Partridge TA, Jaiswal JK, Hoffman EP: A long-read RNA-seq approach to identify novel transcripts of very large genes. Genome 
Res 2020, 30(6):885-897.

30. Ali A, Thorgaard GH, Salem M: PacBio Iso-Seq Improves the Rainbow Trout Genome Annotation and Identifies Alternative Splicing Associated With Economically Important Phenotypes. Front Genet 2021, 12:683408.

31. Singh P, Alley TL, Wright SM, Kamdar S, Schott W, Wilpan RY, Mills KD, Graber JH: Global changes in processing of mRNA 3' untranslated regions characterize clinically distinct cancer subtypes. Cancer Res 2009, 69(24):9422-9430.

32. Pardo-Palacios F, Reese F, Carbonell-Sala S, Diekhans M, Liang C, Wang D, Williams B, Adams M, Behera A, Lagarde J: Systematic assessment of long-read RNA-seq methods for transcript identification and quantification. 2021.

33. Doetschman TC, Eistetter H, Katz M, Schmidt W, Kemler R: The in vitro development of blastocystderived embryonic stem cell lines: formation of visceral yolk sac, blood islands and myocardium. J Embryol Exp Morphol 1985, 87:27-45.

34. Takahashi K, Yamanaka S: Induction of pluripotent stem cells from mouse embryonic and adult fibroblast cultures by defined factors. Cell 2006, 126(4):663-676.

35. Gabut M, Samavarchi-Tehrani P, Wang X, Slobodeniuc V, O'Hanlon D, Sung HK, Alvarez M, Talukder S, Pan Q, Mazzoni EO et al: An alternative splicing switch regulates embryonic stem cell pluripotency and reprogramming. Cell 2011, 147(1):132-146.

36. De Kumar B, Parrish ME, Slaughter BD, Unruh JR, Gogol M, Seidel C, Paulson A, Li H, Gaudenz K, Peak A et al: Analysis of dynamic changes in retinoid-induced transcription and epigenetic profiles of murine Hox clusters in ES cells. Genome Res 2015, 25(8):1229-1243.

37. Agosto LM, Lynch KW: Alternative pre-mRNA splicing switch controls hESC pluripotency and differentiation. Genes Dev 2018, 32(17-18):1103-1104.

38. Revil T, Gaffney D, Dias C, Majewski J, Jerome-Majewska LA: Alternative splicing is frequent during early embryonic development in mouse. BMC Genomics 2010, 11:399.

39. Lu X, Zhao ZA, Wang X, Zhang X, Zhai Y, Deng W, Yi Z, Li L: Whole-transcriptome splicing profiling of E7.5 mouse primary germ layers reveals frequent alternative promoter usage during mouse early embryogenesis. Biol Open 2018, 7(3).

40. Xing Y, Yang W, Liu G, Cui X, Meng H, Zhao H, Zhao X, Li J, Liu Z, Zhang MQ et al: Dynamic Alternative Splicing During Mouse Preimplantation Embryo Development. Front Bioeng Biotechnol 2020, 8:35.

41. Qiao Y, Ren C, Huang S, Yuan J, Liu X, Fan J, Lin J, Wu S, Chen Q, Bo X et al: High-resolution annotation of the mouse preimplantation embryo transcriptome using long-read sequencing. Nat Commun 2020, 11(1):2653.

42. Xu J, Wang H, Liang T, Cai X, Rao X, Huang Z, Sheng G: Retinoic acid promotes neural conversion of mouse embryonic stem cells in adherent monoculture. Mol Biol Rep 2012, 39(2):789-795.

43. Strickland S, Mahdavi V: The induction of differentiation in teratocarcinoma stem cells by retinoic acid. Cell 1978, 15(2):393-403. 
44. Leung SK, Jeffries AR, Castanho I, Jordan BT, Moore K, Davies JP, Dempster EL, Bray NJ, O'Neill P, Tseng $\mathrm{E}$ et al: Full-length transcript sequencing of human and mouse cerebral cortex identifies widespread isoform diversity and alternative splicing. Cell Rep 2021, 37(7):110022.

45. Anders S, Reyes A, Huber W: Detecting differential usage of exons from RNA-seq data. Genome Res 2012, 22(10):2008-2017.

46. Jin L, Chen Y, Crossman DK, Datta A, Vu T, Mobley JA, Basu MK, Scarduzio M, Wang H, Chang C et al: STRAP regulates alternative splicing fidelity during lineage commitment of mouse embryonic stem cells. Nat Commun 2020, 11(1):5941.

47. Laursen KB, Kashyap V, Scandura J, Gudas LJ: An alternative retinoic acid-responsive Stra6 promoter regulated in response to retinol deficiency. J Biol Chem 2015, 290(7):4356-4366.

48. Yang Y, Xiong J, Wang J, Ruan Y, Zhang J, Tian Y, Liu L, Cheng Y, Wang X, Xu Y et al: Novel alternative splicing variants of Klf4 display different capacities for self-renewal and pluripotency in mouse embryonic stem cells. Biochem Biophys Res Commun 2020, 532(3):377-384.

49. Salomonis N, Schlieve CR, Pereira L, Wahlquist C, Colas A, Zambon AC, Vranizan K, Spindler MJ, Pico $A R$, Cline $M S$ et al: Alternative splicing regulates mouse embryonic stem cell pluripotency and differentiation. Proc Natl Acad Sci U S A 2010, 107(23):10514-10519.

50. van der Kant R, Goldstein LS: Cellular functions of the amyloid precursor protein from development to dementia. Dev Cell 2015, 32(4):502-515.

51. Kaji K, Caballero IM, MacLeod R, Nichols J, Wilson VA, Hendrich B: The NuRD component Mbd3 is required for pluripotency of embryonic stem cells. Nat Cell Biol 2006, 8(3):285-292.

52. Hendrich B, Guy J, Ramsahoye B, Wilson VA, Bird A: Closely related proteins MBD2 and MBD3 play distinctive but interacting roles in mouse development. Genes Dev 2001, 15(6):710-723.

53. Ee LS, McCannell KN, Tang Y, Fernandes N, Hardy WR, Green MR, Chu F, Fazzio TG: An Embryonic Stem Cell-Specific NuRD Complex Functions through Interaction with WDR5. Stem Cell Reports 2017, 8(6):1488-1496.

54. Kent WJ, Sugnet CW, Furey TS, Roskin KM, Pringle TH, Zahler AM, Haussler D: The human genome browser at UCSC. Genome Res 2002, 12(6):996-1006.

55. Thorvaldsdóttir H, Robinson JT, Mesirov JP: Integrative Genomics Viewer (IGV): high-performance genomics data visualization and exploration. Brief Bioinform 2013, 14(2):178-192.

56. Kohen R, Barlev J, Hornung G, Stelzer G, Feldmesser E, Kogan K, Safran M, Leshkowitz D: UTAP: Userfriendly Transcriptome Analysis Pipeline. BMC Bioinformatics 2019, 20(1):154.

57. Chen $\mathrm{C}$, Khaleel SS, Huang $\mathrm{H}, \mathrm{Wu} \mathrm{CH}$ : Software for pre-processing Illumina next-generation sequencing short read sequences. Source Code Biol Med 2014, 9:8.

58. Dobin A, Davis CA, Schlesinger F, Drenkow J, Zaleski C, Jha S, Batut P, Chaisson M, Gingeras TR: STAR: ultrafast universal RNA-seq aligner. Bioinformatics 2013, 29(1):15-21.

59. Anders S, Pyl PT, Huber W: HTSeq-a Python framework to work with high-throughput sequencing data. Bioinformatics 2015, 31(2):166-169. 
60. R Development Core Team: R: A language and environment for statistical computing. R Foundation for Statistical Computing. In. Vienna, Austria: ISBN 3-900051-07-0; 2008.

61. Love MI, Huber W, Anders S: Moderated estimation of fold change and dispersion for RNA-seq data with DESeq2. Genome Biol 2014, 15(12):550.

62. Wang L, Wang S, Li W: RSeQC: quality control of RNA-seq experiments. Bioinformatics 2012, 28(16):2184-2185.

63. De Coster W, D'Hert S, Schultz DT, Cruts M, Van Broeckhoven C: NanoPack: visualizing and processing long-read sequencing data. Bioinformatics 2018, 34(15):2666-2669.

64. Quinlan AR, Hall IM: BEDTools: a flexible suite of utilities for comparing genomic features. Bioinformatics 2010, 26(6):841-842.

65. Lawrence $M$, Huber W, Pagès $H$, Aboyoun $P$, Carlson $M$, Gentleman R, Morgan MT, Carey VJ: Software for computing and annotating genomic ranges. PLoS Comput Biol 2013, 9(8):e1003118.

66. Frazee AC, Pertea G, Jaffe AE, Langmead B, Salzberg SL, Leek JT: Ballgown bridges the gap between transcriptome assembly and expression analysis. Nat Biotechnol 2015, 33(3):243-246.

67. Gal-Oz ST, Haiat N, Eliyahu D, Shani G, Shay T: DochaP: the domain change presenter. Nucleic Acids Res 2021, 49(W1):W162-W168.

68. El-Gebali S, Mistry J, Bateman A, Eddy SR, Luciani A, Potter SC, Qureshi M, Richardson LJ, Salazar GA, Smart A et al: The Pfam protein families database in 2019. Nucleic Acids Res 2019, 47(D1):D427-D432.

\section{Tables}

Table 1 is available in the Supplemental Files section.

\section{Figures}




\section{Figure 1}

\section{A}

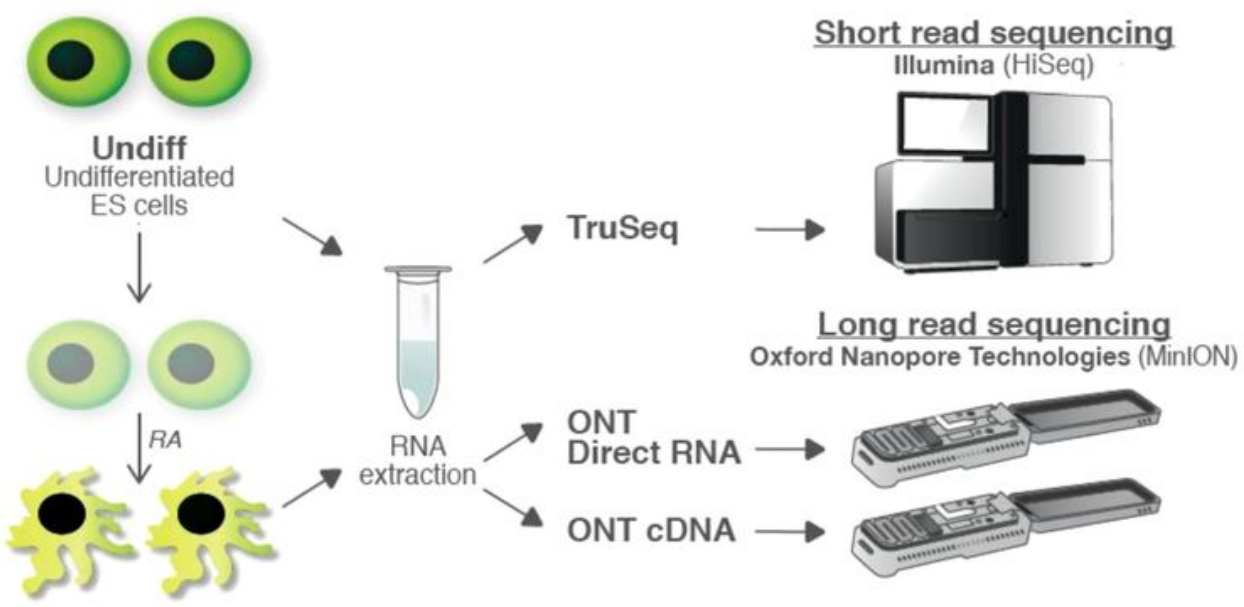

Day 4

Differentiated ES cells

B

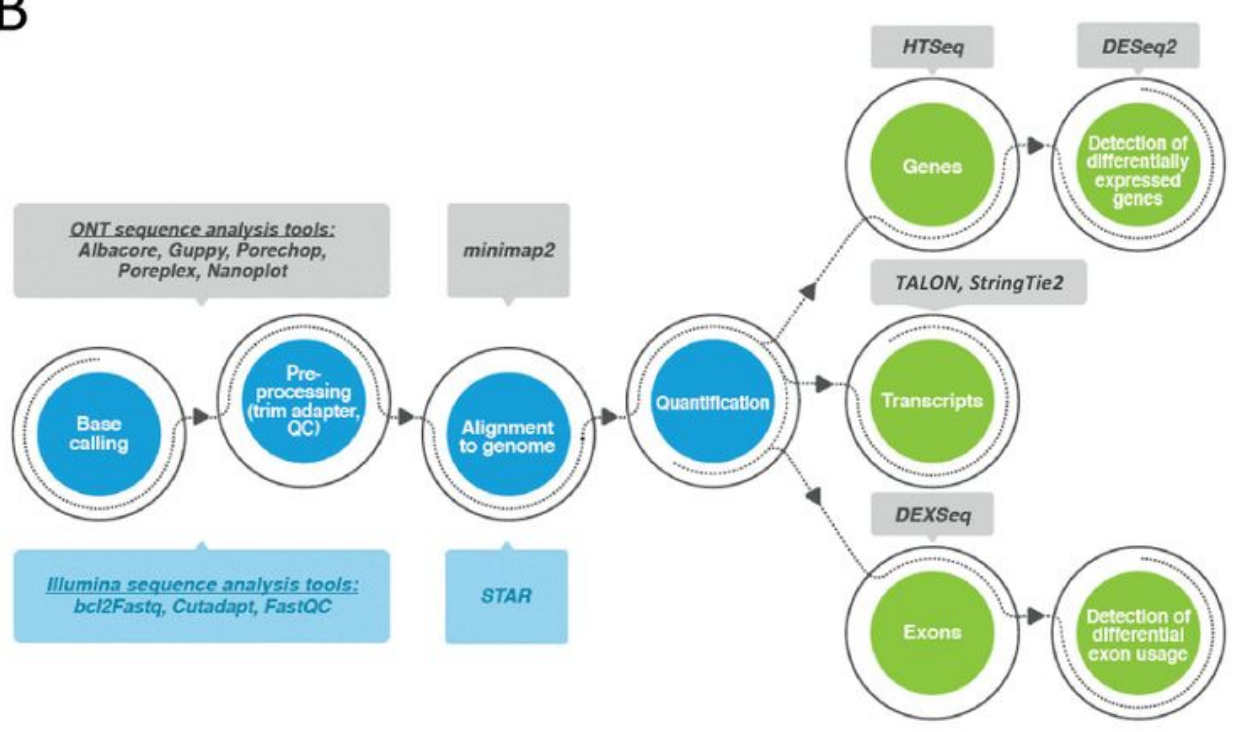

\section{Figure 1}

(A) A schematic workflow of the experimental design. Mouse embryonic stem cells were grown for four days in mES growth medium ("Undiff"), and then for an additional eight days, of them four days with retinoic acid (RA) ("Day4"). RNA was extracted and sequencing libraries were prepared for short-read sequencing with Illumina (TruSeq library), and long-read sequencing with Oxford Nanopore Technologies, either CDNA or Direct RNA library platforms. 
(B) The bioinformatics analysis pipeline. Raw sequences were pre-processed and aligned to the mouse genome using tools for either short or long reads. Expression was quantified at gene, transcript and exon levels, to identify differentially expressed genes (DEGs) and differential exon usages (DEUs) in Undiff versus Day4 samples, using the same tools for all platforms.

\section{Figure 2}

A

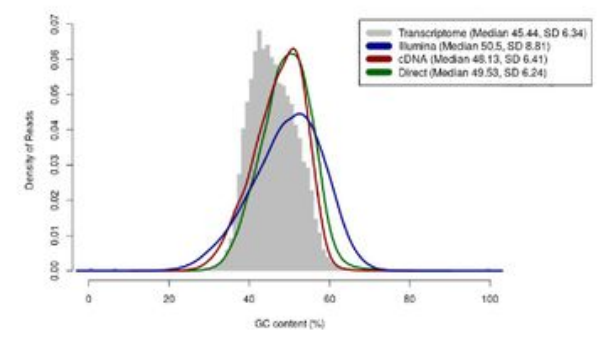

B
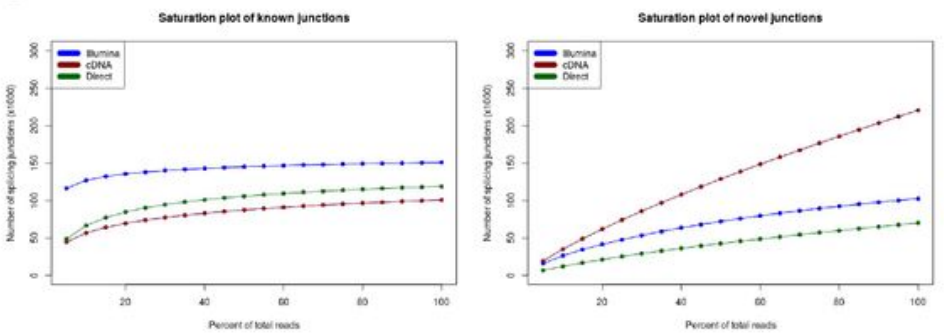

C

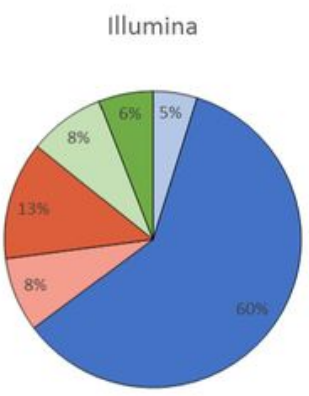

$\square$ Annotated (exactly 1 read) $\square$ Partial novel (exactly 1 read) $\square$ Complete novel (exactly 1 read)
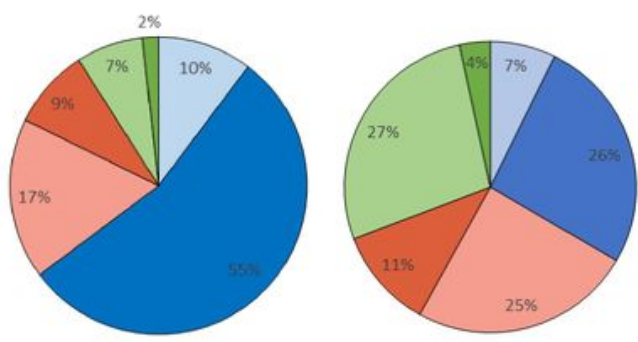

Annotated (at least 2 reads)

- Partial novel (at least 2 reads)

口Complete novel (at least 2 reads)

D

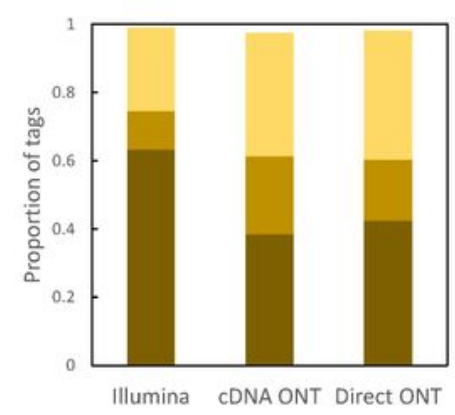

$\mathrm{E}$

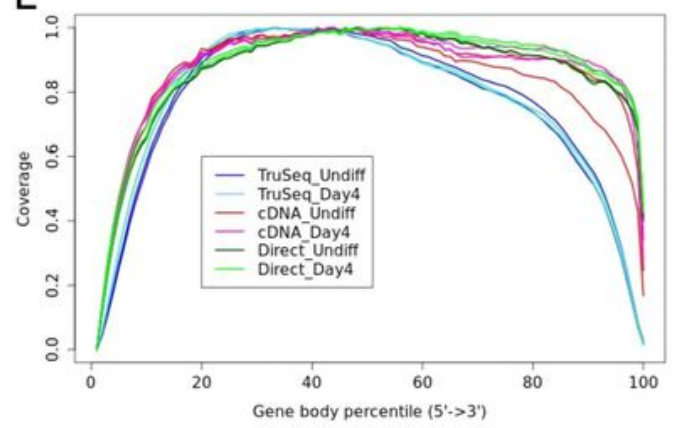

Figure 2 
Comparison of aligned read quality features between Illumina TruSeq, ONT cDNA and ONT Direct RNA platforms. Panels A-D relate to averaged replicates of Day4 datasets.

(A) GC content distribution of reads across the platforms. In grey is the theoretical distribution calculated for all transcripts ("Transcriptome"). Median and standard deviation (SD) are noted in the legend.

(B) Saturation of known and novel splice junctions. The plot reveals saturation by resampling $5 \%, 10 \%$, $15 \%$, etc. of the total alignments.

(C) The proportions of reads, detected in one of the six categories of junction annotations, supported by a single read, or by at least two reads, and defined as Known (both 5' and $3^{\prime}$ splice sites are annotated by reference gene models), Partial novel (one of the splice sites $5^{\prime}$ or $3^{\prime}$ is novel), or Complete novel (both splice sites $5^{\prime}$ and $3^{\prime}$ are novel).

(D) Proportion of tags over exonic features. Tags were defined by RSeQC (a read spliced once is counted as two tags), all tags were normalized to "Tags/Kb".

(E) Profile plot of gene body coverage depicting the proportion of coverage throughout the gene body, across platforms and differentiation days. 
Figure 3
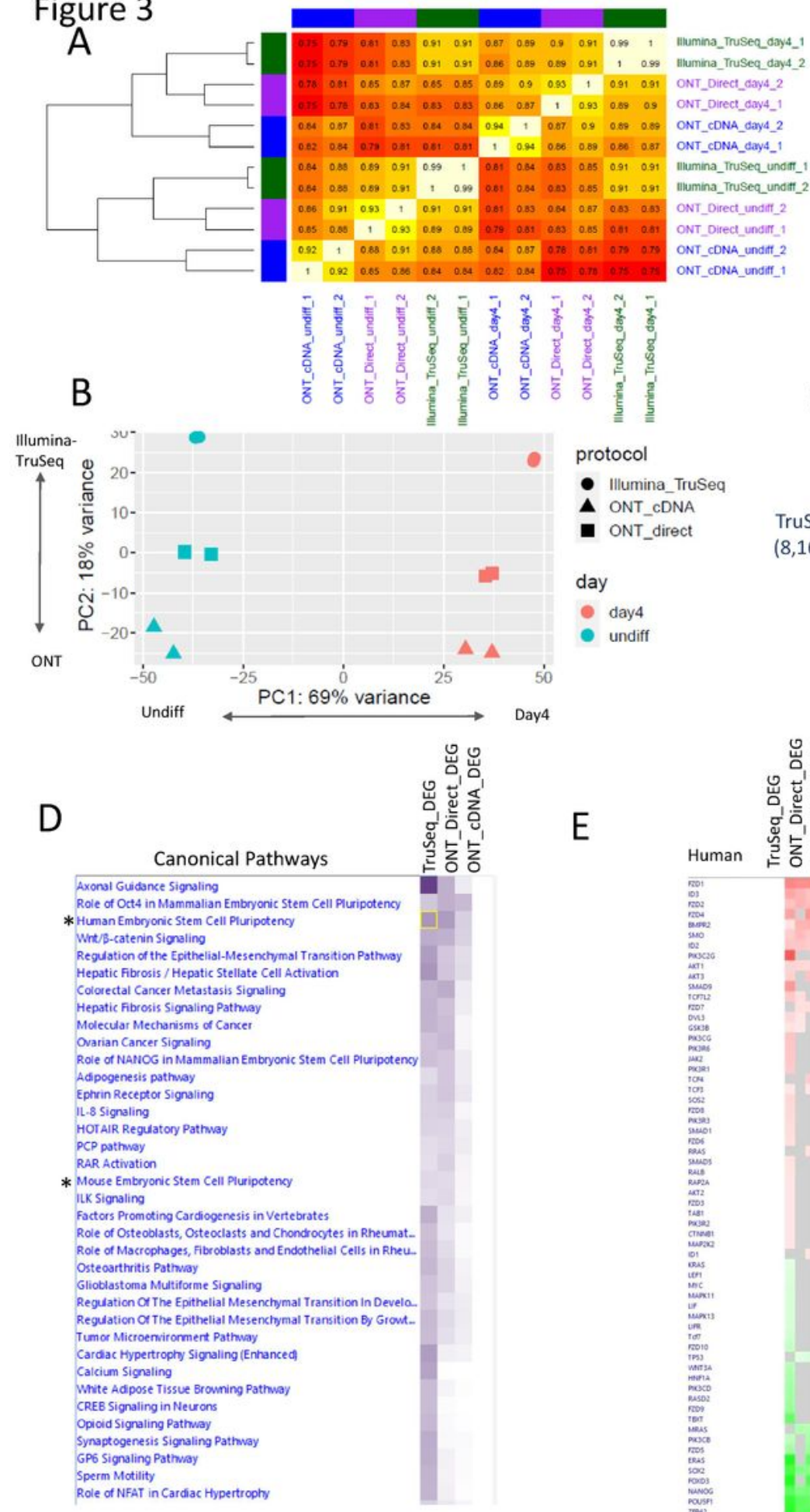

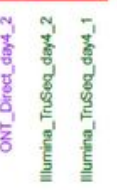

protocol

- Illumina_TruSeq

- ONT_cDNA

- ONT_direct

day

- day4

undiff

$\mathrm{E}$

\section{C}

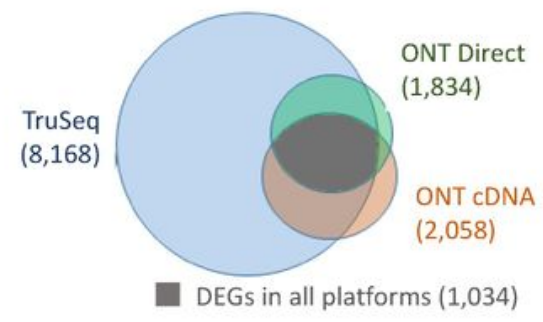

ONT Direct

$(1,834)$

ONT cDNA

,058)

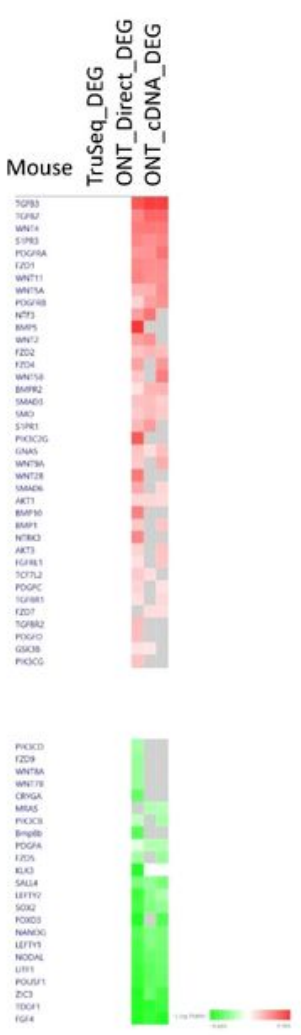

\section{Figure 3}

Gene-level expression analysis across the sequencing platforms.

(A) Heatmap of Spearman correlation coefficients of raw gene counts for the Day4 and Undiff samples (in duplicates), sequenced with either Illumina TruSeq, ONT cDNA or Direct RNA. 
(B) Principal component analysis (PCA) of log-normalized values (rld) from all samples. Colours denote differentiation day and shapes denote sequencing platform.

(C) Proportional Venn diagram depicting the number of DEGs and their overlap, detected by the different sequencing platform.

(D) Ingenuity pathway analysis showing the top enriched canonical pathways (z-score), computed using DEGs from the three sequencing platforms. $\left(^{*}\right)$ indicates pathways elaborated in $(E)$.

(E) Ingenuity pathway analysis showing the log-fold-change expression of the top up-and downregulated DEGs for the human or mouse embryonic stem cell pluripotency pathways. 


\section{Figure 4}

A

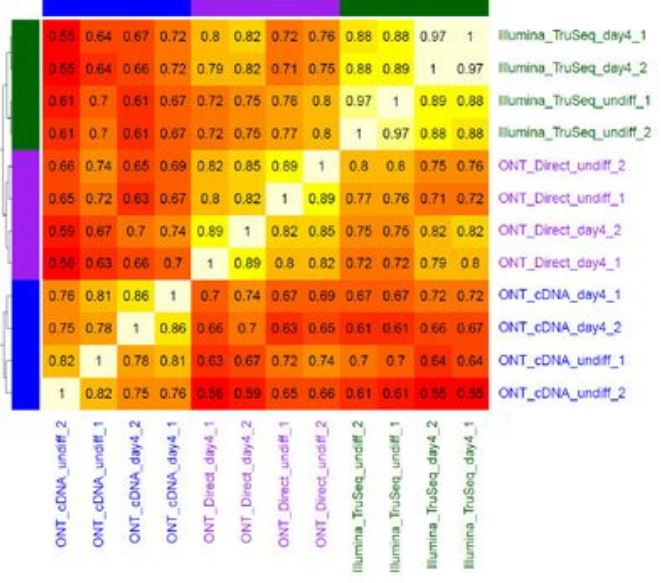

B
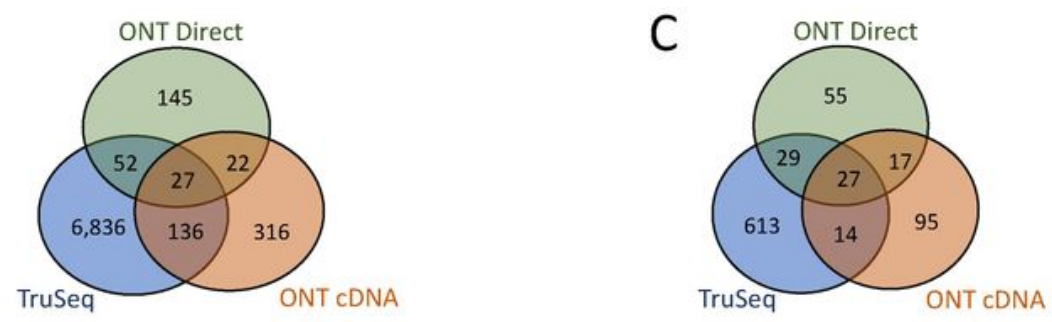

D

All DEUs (7534)

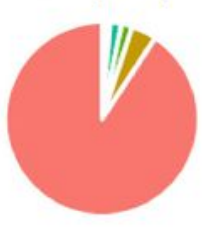

III_TruSeq [90.7\%]

ONT_CDNA [4.2\%]

- ONT_CDNA \& III_TruSeq [1.8\%]

ONT_Direct [1.9\%]

ONT_Direct \& III_Truseq [0.7\%]

- ONT_Direct \& ONT_CDNA [0.3\%]

- ONT_Direct \& ONT_CDNA \& III_TruSeq [0.4\%]

3' UTR exons (520)

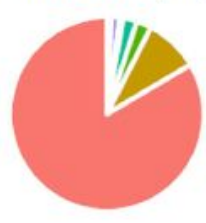

III_TruSeq [83.5\%]

ONT_CDNA [9.0\%]

ONT_CDNA \& III_TruSeq [2.5\%]

ONT_Direct [2.3\%]

ONT_Direct \& III_TruSeq [0.6\%]

ONT_Direct \& ONT_CDNA [1.3\%]

ONT_Direct \& ONT_CDNA \& III_TruSeq [0.8\%]

E

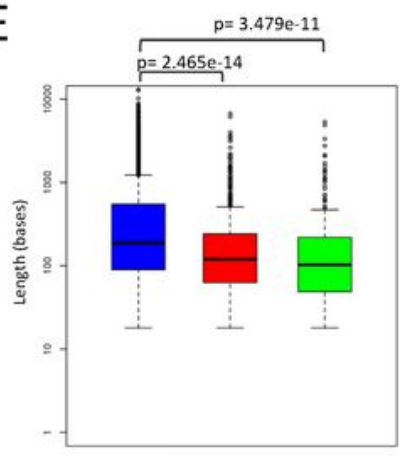

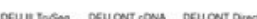

$\mathrm{F}$

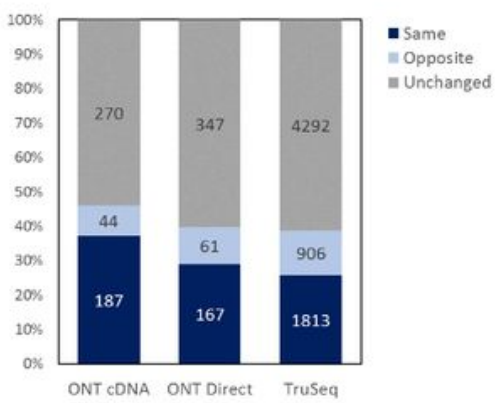

Figure 4

Exon-level expression analysis across the sequencing platforms.

(A) Heatmap of Spearman correlation coefficients of raw exon counts for the Day4 and Undiff samples (in duplicates), sequenced with either Illumina TruSeq, or with ONT cDNA or Direct RNA. 
(B) Venn diagram depicting the overlap of DEUs detected in the different sequencing platforms.

(C) Venn diagram depicting the overlap of DEUs, accounting only for exons which passed the expression filtering cutoffs in all platforms (see Methods).

(D) Proportions of DEUs detected by the various platforms (left, a pie presentation of the Venn diagram presented in (B)), and specifically for DEUs in genes 3' UTR (right).

(E) Box plot distribution of DEU lengths (in bases) per platform. Kolmogorov-Smirnov test p-values are shown.

(F) Proportions calculated by a comparison of DEUs to DEGs, categorized by the gene: same significant trend ("same", both upregulated or downregulated); opposite significant trend, ("opposite"); or the gene did not change significantly ("unchanged"). Proportions are shown for each sequencing platform separately, depicting the number of DEUs. 
Figure 5

A

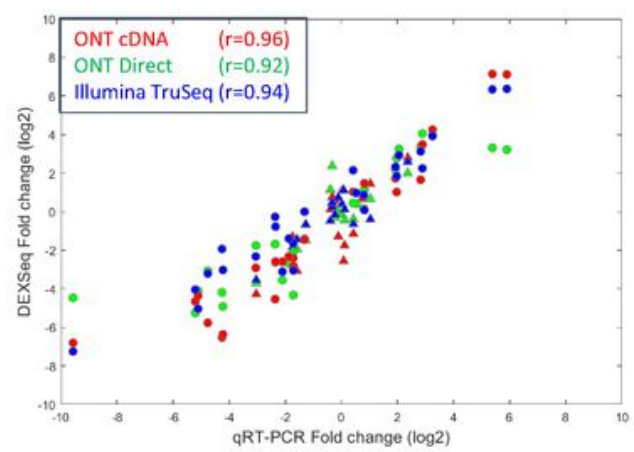

B
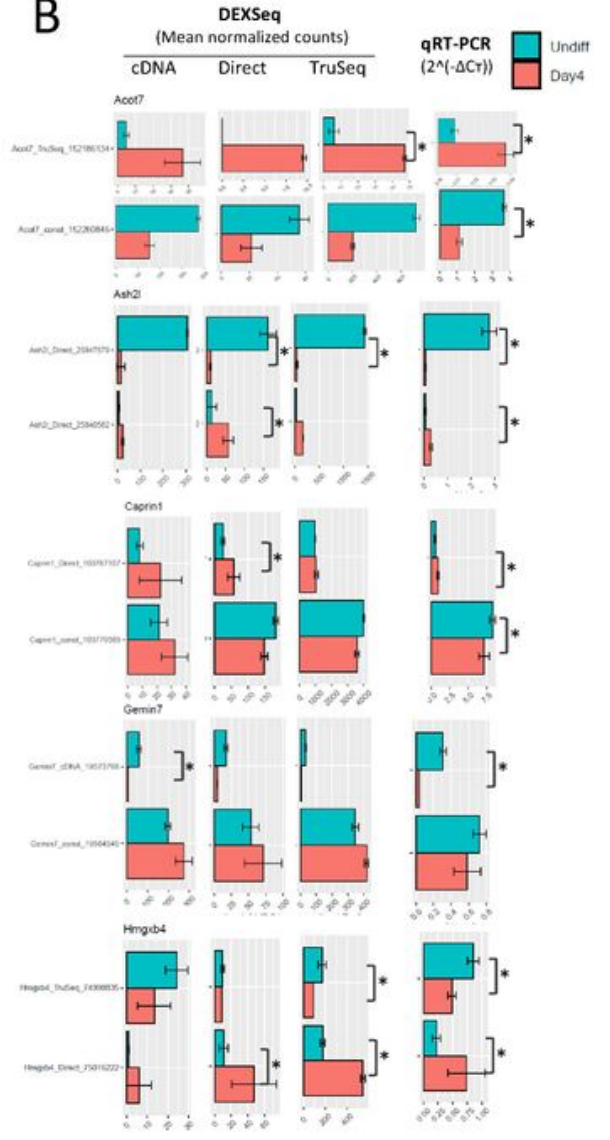
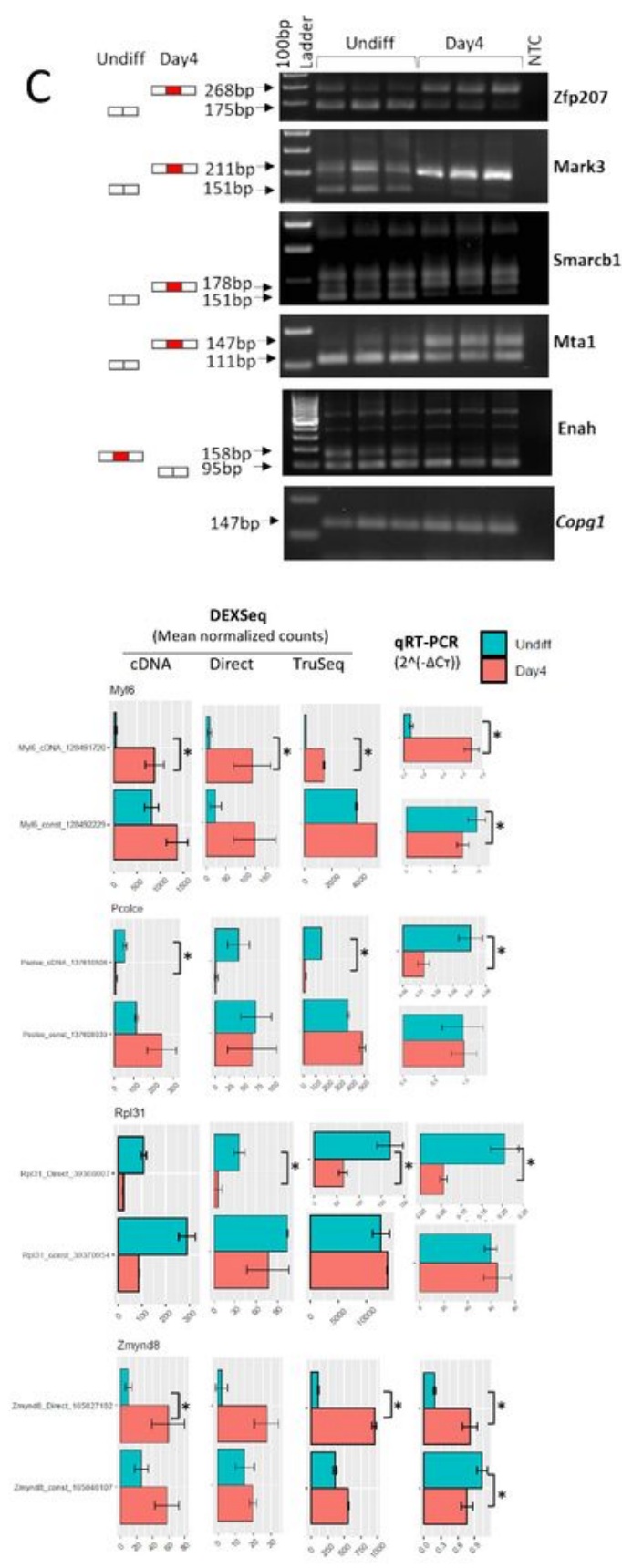

\section{Figure 5}

Validation of selected DEUs.

(A) Scatter plot of the fold change expression ratio (Day4 versus Undiff samples) of 42 selected exons, as quantified by either qRT-PCR or DEXSeq analysis of each platform. Values represent log2 fold-change expression between the mean Day4 values and the mean Undiff values (for qRT-PCR, $\left(2^{\wedge}(-\Delta C T)\right.$; for 
DEXSeq, normalized exon counts). Constitutive exons are depicted in triangles, whereas DEUs are in circles. Spearman correlation coefficients between qRT-PCR and DEXSeq log2 fold-change values are denoted.

(B) qRT-PCR validation of DEUs from 11 genes. Computed DEXSeq mean normalized counts (with error bars) are shown for each validated exon in each platform, along with its mean qRT-PCR $2^{\wedge}(-\triangle C T)$ values (five replicates for each day, depicted by error bars). More than one exon is shown per gene, either a DEU and a constitutive exon, or two DEUs. (*) Significant changes in qRT-PCR (adjusted P-value $=<0.05$ ) between the days, or a significant DEU by DEXSeq analysis.

(C) Validation by RT-PCR for five genes with DEU events, shown in triplicates per differentiation day. The expected products with their sizes are shown in the left panel, the alternative intronic exons are depicted in red and constitutive exons in white, arranged according to the predicted differentiation state expression preference. NTC is a negative control, Copg1 is the loading control gene. 


\section{Figure 6}

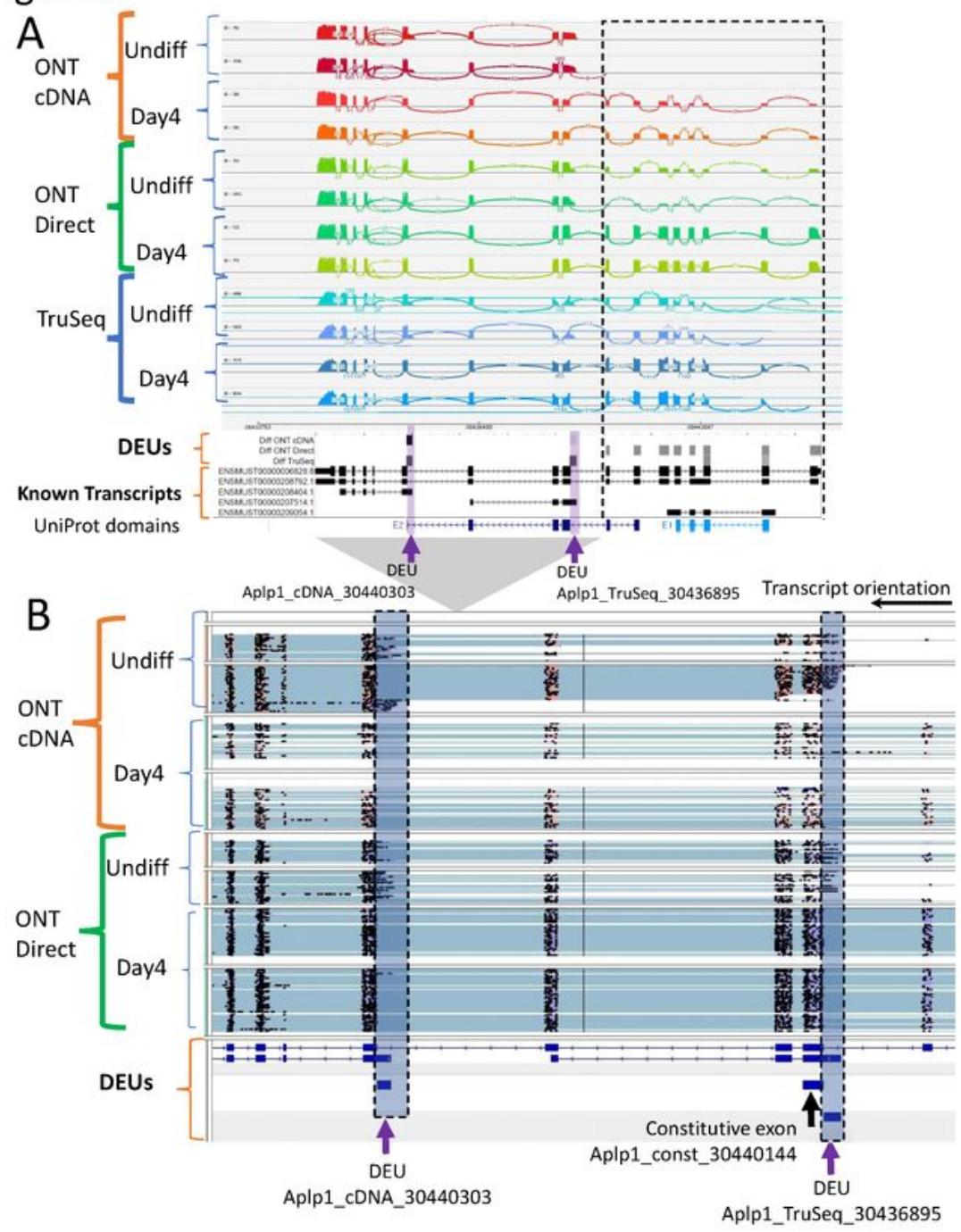

C

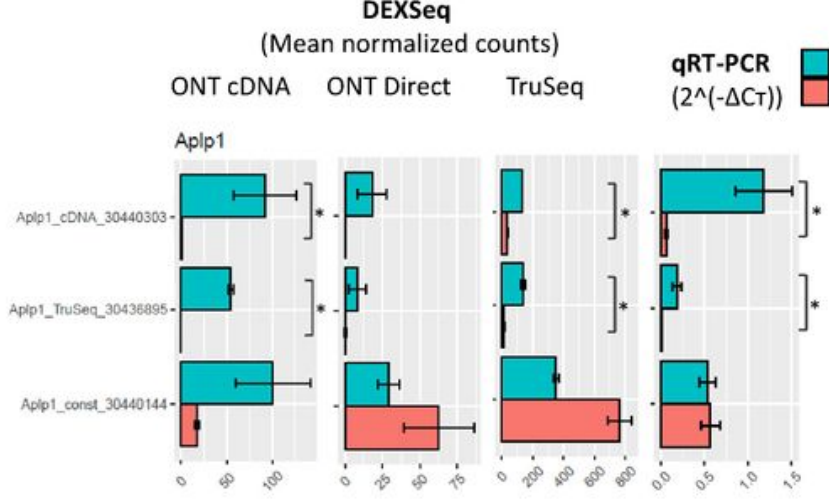

\section{Figure 6}

DEU analysis for the amyloid precursor-like protein 1 (Aplp1) gene.

(A) Sashimi plot visualization of read coverage and splice junctions along the entire Aplp1 gene. Bottom tracks show DEU and known transcripts (GENCODE). In the DEU track, the grayscale is indicative of fold 
change (Day4 versus Undiff). The area in the rectangle marks the gene $5^{\prime}$-end, containing many DEUs. Purple arrows denote the DEUs selected for validation.

(B) Visualization of ONT reads spanning the 3'-end of the Aplp1 gene. Purple arrows denote the exons selected for validation.

(C) Validation of Aplp1 DEUs by qRT-PCR. DEXSeq exon counts as explained in Fig. 5.

\section{A Figure 7}

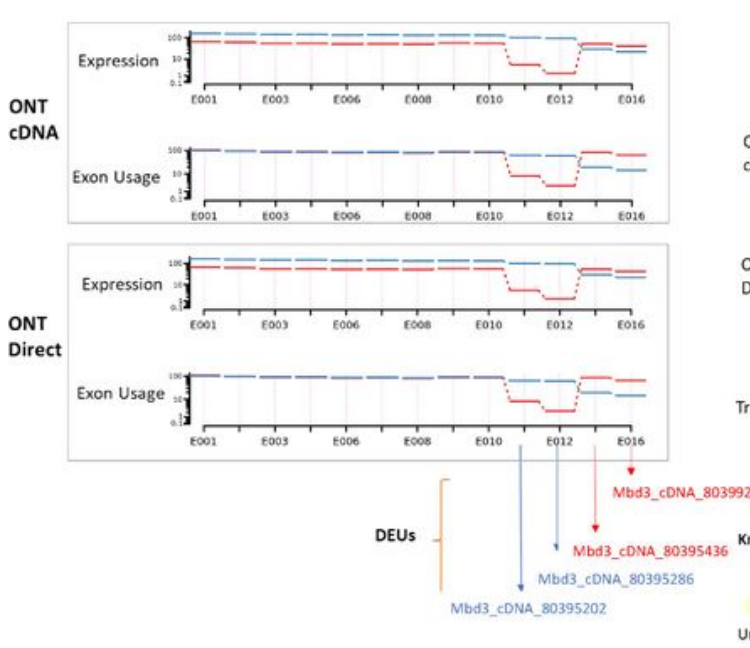

\section{B}

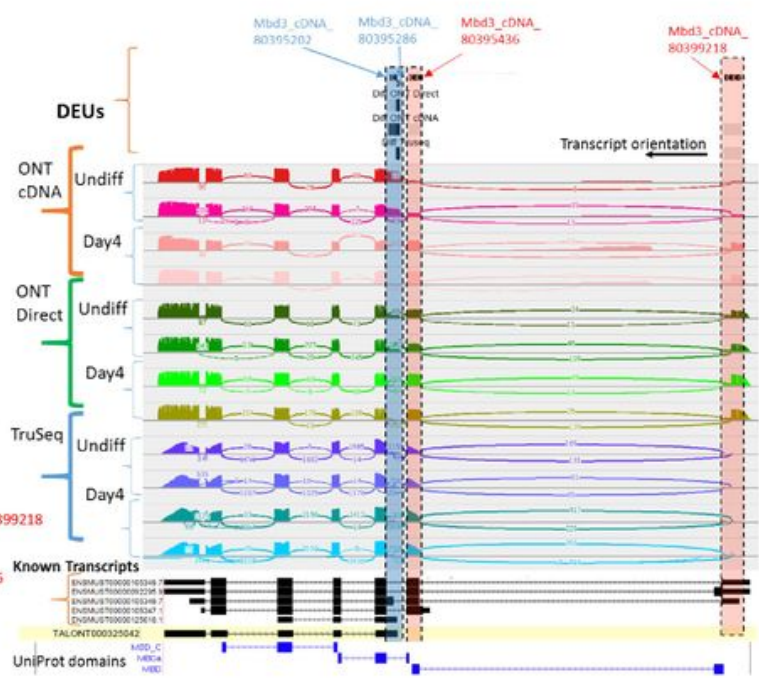

DEXSeq

C
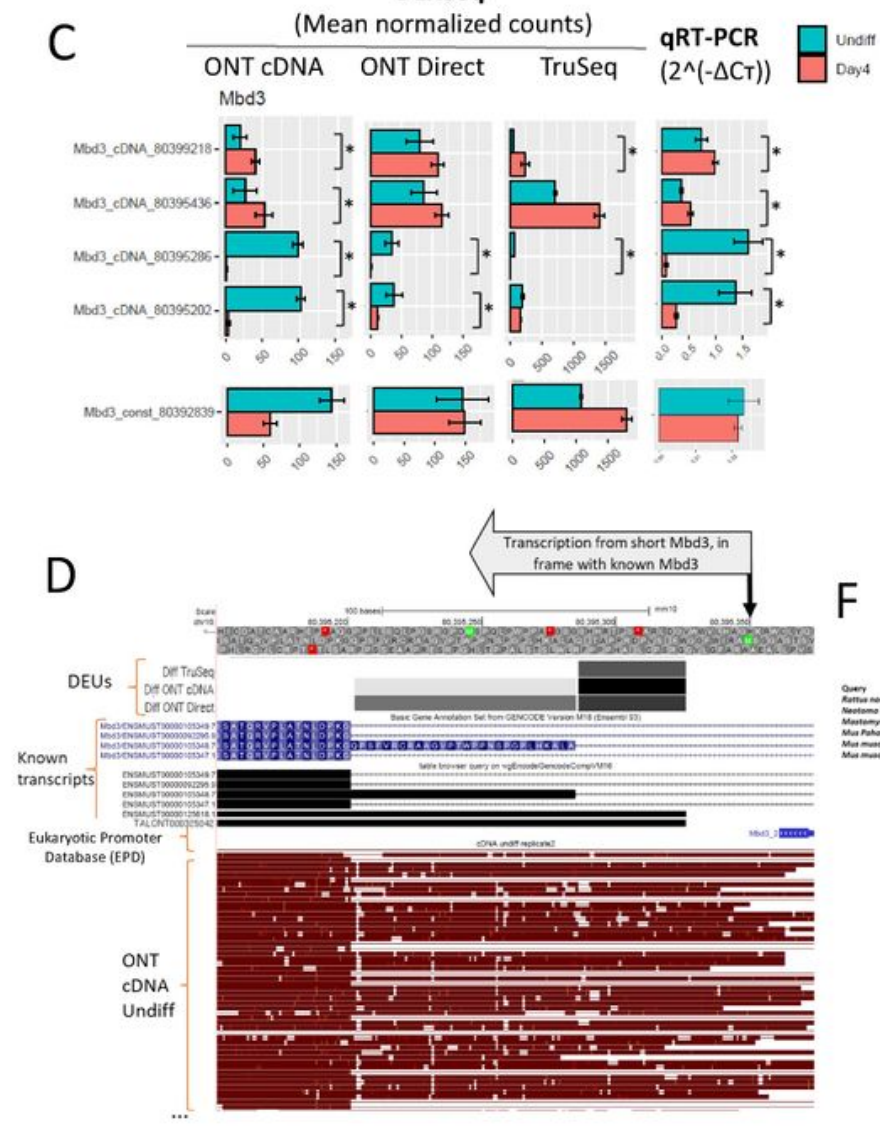

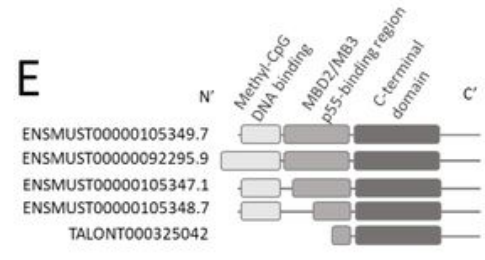

$\mathrm{F}$

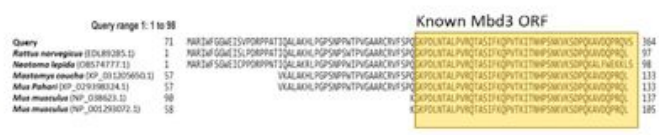




\section{Figure 7}

DEU analysis for the methyl-CpG binding domain protein 3 (Mbd3) gene.

(A) DEXSeq results for visualization of exon usage and estimated expression of Mbd3 gene exons, for Day4 (red) and Undiff (blue) samples, sequenced by ONT cDNA and Direct RNA platforms. The arrows denote the DEUs selected for validation.

(B) Sashimi plot visualization of read coverage and splice junctions along the Mbd3 gene. Top track: Predicted DEUs. Bottom track: known Mbd3 transcript models, and a novel transcript predicted by the TALON pipeline.

(C) Validation of Mbd3 DEUs by qRT-PCR, as explained in Fig. 5.

(D) Schematic domain architecture of the protein products of either known or predicted TALON transcripts. Pfam domains from left to right: Methyl-CpG binding domain (PF01429), MBD2/MB3_p55 binding domain (PF16564), and C-terminal domain of methyl-CpG binding protein 2 and 3 (PF14048).

(E) Top tracks show the alternative promoter and DEUs. Subsets of ONT cDNA Undiff reads starting approximately at the alternative transcription start site (TSS), which may encode for an ORF starting at the depicted methionine (see top arrow pointing downwards). In the DEU track, the grayscale is indicative of fold change (Day4 versus Undiff). A plot of all ONT cDNA reads is shown in Supplementary Fig. 9.

(F) Multiple Sequence alignment of the protein sequence encoded by the alternative Mbd3 TSS, starting at the above depicted methionine (E), with orthologue protein sequences.

\section{Supplementary Files}

This is a list of supplementary files associated with this preprint. Click to download.

- Table1.xlsx

- TableS1.xlsx

- Tables2.xIsx

- TableS3.xIsx

- Tables4.xlsx

- TableS5A.xlsx

- Tables5B.xlsx

- Tables6.pdf

- SupplementaryFile127222.pdf

- Supplementalfigures.pdf 